Reviews

\title{
Infrared Spectroscopy-Mid-infrared, Near-infrared, and Far-infrared/Terahertz Spectroscopy
}

\author{
Yukihiro OZAKI ${ }^{* * * *}$ \\ *School of Biological and Environmental Sciences, Kwansei Gakuin University, Sanda, Hyogo 669-1337, Japan \\ **Toyota Physical and Chemical Research Institute, Nagakute, Aichi 480-1192, Japan
}

\begin{abstract}
This article aims to overview infrared (IR) spectroscopy. Simultaneously, it outlines mid-infrared (MIR), near-infrared (NIR), and far-infrared (FIR) or terahertz (THz) spectroscopy separately, and compares them in terms of principles, characteristics, advantages, and applications. MIR spectroscopy is the central spectroscopic technique in the IR region, and is mainly concerned with the fundamentals of molecular vibrations. NIR spectroscopy incorporates both electronic and vibrational spectroscopy; however, in this review, I have chiefly discussed vibrational NIR spectroscopy, where bands due to overtones and combination modes appear. FIR or THz spectroscopy contains both vibrational and rotational spectroscopy. However, only vibrational FIR or THz spectroscopy has been discussed in this review. These three spectroscopy cover wide areas in their applications, making it rather difficult to describe these various topics simultaneously. Hence, I have selected three key topics: hydrogen bond studies, applications of quantum chemical calculations, and imaging. The perspective of the three spectroscopy has been discussed in the last section.
\end{abstract}

Keywords Terahertz, near-infrared, infrared, far-infrared, imaging, quantum chemical calculations, hydrogen bonds

(Received December 17, 2020; Accepted February 11, 2021; Advance Publication Released Online by J-STAGE February 19, 2021)

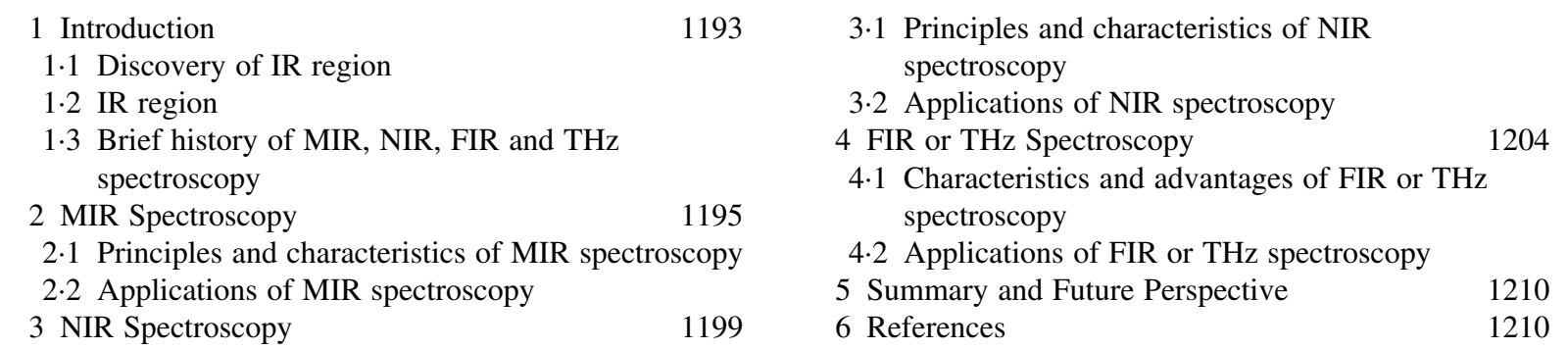

\section{Introduction}

The infrared (IR) region $\left(800 \mathrm{~nm}-1 \mathrm{~mm}, 12500-10 \mathrm{~cm}^{-1}\right)$ is wide in terms of energy; thus, it is impossible to treat spectroscopy in the entire IR region as a single spectroscopy. Therefore, mid-infrared (MIR), ${ }^{1-4}$ near-infrared (NIR), ${ }^{1,5-7}$ and far-infrared (FIR) ${ }^{1,8,9}$ spectroscopy have been developed independently over the years, although the development of NIR and FIR spectroscopy has been far behind that of MIR spectroscopy. However, in the last three decades, remarkable development has been made in NIR spectroscopy. ${ }^{5-7}$ Terahertz $(\mathrm{THz})$ spectroscopy was initially performed at the end of the 1990s. ${ }^{10-12}$ Thus, it is time to overview the three spectroscopy and compare them. It is difficult to find a review that outlines the spectroscopy in the entire IR region. Therefore, this attempt at overviewing the IR spectroscopic techniques is challenging, yet crucial. This overview will provide insights into molecular

E-mail: yukiz89016@gmail.com vibrations, including fundamentals, overtones, and combinations, as well as intermolecular and lattice vibrations, thereby

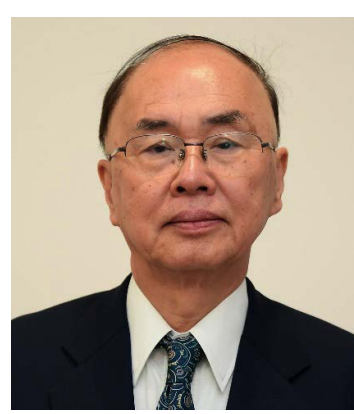

Yukihiro OzaKI received his $\mathrm{Ph} . \mathrm{D}$, in 1978 from Osaka University. He joined Kwansei Gakuin University in 1989. Since 1993 he was a professor in School of Science and Technology until March, 2018. Currently, Ozaki is a professor emeritus of the university and a guest professor or a researcher at Kobe University, Riken, Toyota Physical and Chemical Research Institute. He has been involved in studies of a wide range of molecular spectroscopy, covering from far-ultraviolet to far-infrared/Terahertz spectroscopy and also Raman spectroscopy. He has been a Fellow of Royal Society of Chemistry. He received several awards including, the Spectroscopical Society of Japan Award (2002), The Japan Society for Analytical Chemistry Award (2008), Chemical Society of Japan Award (2017), The Medal with Purple Ribbon (2018), and Pittsburg Spectroscopy Award (2019), Charles Mann Award for Applied Raman Spectroscopy (2020). 


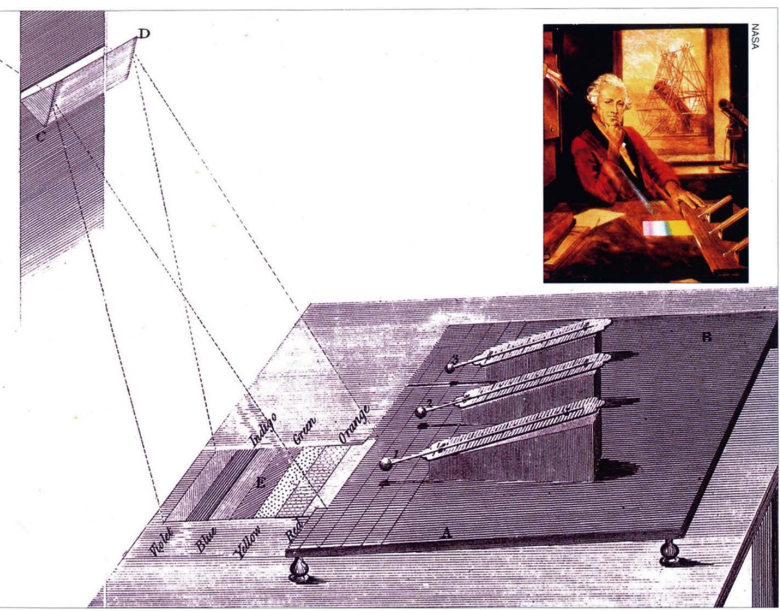

Fig. 1 The portrait of William Herschel and his experimental set up in 1800 .

deepening the understanding of molecular vibrations. MIR, NIR, and FIR or THz spectroscopy are not three sisters; rather MIR is the mother of NIR spectroscopy because the overtones and combinations originate from the fundamentals. Notably, electronic and rotational spectra are also observed in the IR region. Thus, it should be noted that IR spectroscopy is mostly a vibrational spectroscopy; however, it is concerned with electronic and rotational spectroscopy as well.

One of the objectives of this tutorial review is to describe the principles, characteristics, and advantages of MIR, NIR, and FIR or THz spectroscopy, and then, compare them. To compare their characteristics and advantages in this review, I mainly discuss hydrogen bonding studies, quantum chemical calculations, and spectroscopic imaging. All spectroscopy (MIR, NIR, and FIR or THz) are useful for the investigation of hydrogen bonds; however, each method has its own advantages. Quantum chemical calculations have recently been used for the three spectroscopy; however, in quantum chemistry, their strategies and tactics are considerably different. MIR, NIR, and $\mathrm{THz}$ spectroscopy images have their own benefits; thus, they have been used for different purposes. To demonstrate the applications of the three spectroscopy, emphasis has been placed on polymer research as the common research objective because it is of significance for basic research as well as applications including industrial applications. I have also discussed the future perspective of NIR, IR, and FIR or $\mathrm{THz}$ spectroscopy in the concluding section.

\subsection{Discovery of IR region}

An invisible component beyond the red end of the solar spectrum was discovered in 1800 by William Herschel, a German-born British astronomer. Herschel investigated the effect of sunlight on the temperature increase in each colour part separated by a prism. Figure 1 shows a portrait of Herschel and the experimental setup he used. It is likely that he coincidentally observed an evident increase in the temperature beyond the red coloured component. He considered that there exists a different type of invisible radiation from visible light beyond the red end of the sunlight; he named this radiation "heat ray". This was a significant discovery in science; however, even Herschel could not imagine that this was light. After the discovery of "heat ray" numerous scientists, including Herschel's son, explored the heat ray. In 1835, Ampere confirmed that "heat ray" was invisible light, having wavelengths that are longer than that of the visible light. The light with the longer wavelength was named "infrared" (IR). In 1864, Maxwell proved that ultraviolet (UV), visible, and IR light are all electromagnetic waves. In 1888 , Hertz provided the experimental evidence that proved the electromagnetic nature of light.

\subsection{IR region}

The IR light ranges from $800 \mathrm{~nm}\left(12500 \mathrm{~cm}^{-1}\right)$ to $1 \mathrm{~mm}$ $\left(10 \mathrm{~cm}^{-1}\right)$. In terms of energy, it is more than a thousand times (correctly 1250 times) from the shortest wavelength edge to the longest wavelength edge of the IR light. The IR region is extremely large; thus, it is impossible to treat the entire region as one region from the perspective of spectroscopy. Hence, it is divided into three regions: NIR, ${ }^{5-7}$ MIR, ${ }^{2-4}$ and FIR ${ }^{8,9}$ Thus, in this review, "IR" refers to the entire IR region. For the last two decades, $\mathrm{THz}$ spectroscopy has been developed steadily; nowadays, the $\mathrm{THz}$ region is defined as the region extending from 0.1 to $10 \mathrm{THz}$ (333 to $\left.3.3 \mathrm{~cm}^{-1}\right){ }^{10-12}$ Hence, the FIR (400 $10 \mathrm{~cm}^{-1}$ ) and $\mathrm{THz}$ regions overlap with each other. The difference between FIR and THz spectroscopy will be explained in Sect. 4. The FIR and THz regions are the boundary between light and radio waves. ${ }^{8-12}$

The MIR region $\left(2.5-25 \mu \mathrm{m}, 4000-400 \mathrm{~cm}^{-1}\right)$ is the region where the fundamental bands of molecular vibrations are observed. ${ }^{2-4}$ The bands derived from overtones and combinations also appear in the IR region. The NIR region extends from 800 to $2500 \mathrm{~nm}\left(12500\right.$ to $\left.4000 \mathrm{~cm}^{-1}\right)$, and is concerned with both electronic and vibrational spectroscopy. ${ }^{1 c, 5-7}$ In the NIR region, bands, arising from electronic transitions as well as those due to overtones and combinations, are observed. The NIR region is defined as the region where the fundamentals of the vibrational modes are not observed. In the FIR or $\mathrm{THz}$ region, bands due to intermolecular, lattice, and skeleton vibrations appear. ${ }^{8-12}$ There is no clear boundary between the MIR and FIR regions. Note that FIR or $\mathrm{THz}$ spectroscopy is also concerned with rotational spectroscopy, ${ }^{8-12}$ although I will not discuss rotational spectroscopy in this review.

\subsection{Brief history of MIR, NIR, FIR, and THz spectroscopy}

Histories of MIR, NIR, and FIR spectroscopy are described by Sheppard in detail in the Encyclopaedia of Vibrational Spectroscopy (J. M. Chalmers and P. R. Griffiths ed.). ${ }^{13}$

In this review, I briefly discuss their histories and that of THz, which is newer than the rest. In the $1880 \mathrm{~s}$, Abney and Festing systematically measured the MIR spectra of a series of simple organic compounds. They used a bolometer as a detector. ${ }^{13}$ Coblentz was a pioneer in the application of MIR spectroscopy in chemistry. He collected MIR spectra of many compounds. The number reached 130 in 1905 . The relationship between MIR spectra and molecular structure was investigated by many researchers from early 20th century. However, it was after World War II that MIR spectrometers made marked progress, owing to the development of highly sensitive thermocouples and high-quality prisms. In the 1960s, it was a golden age of dispersed MIR spectrometers with grating.

Since the 1970s, Fourier-transform (FT) spectroscopy has been introduced to the MIR spectroscopy. It was truly a revolution of MIR spectroscopy. ${ }^{2-4}$ FT spectroscopy allows high accuracy in determining both the wavenumber and peak intensity, and exhibits high spectral resolution. Moreover, MIR measurement has become much easier. Many new variations of the MIR spectroscopy, such as micro MIR, photoacoustic spectroscopy (PAS), and time-resolved MIR spectroscopy, have become available owing to the introduction of the FT technique 
to the MIR region. ${ }^{2-4}$ Among the recent advancements in MIR spectroscopy, vibrational circular dichroism (VCD), ${ }^{14}$ twodimensional IR (2D-IR),${ }^{15}$ nanoIR,${ }^{16}$ imaging, ${ }^{17,18}$ cascade laserbased MIR spectroscopy, ${ }^{19}$ and MEAIRS $^{20}$ are particularly notable.

The development of NIR spectroscopy was slow at the beginning of the 20th century; however, it is noted that, in the 1930s, spectroscopists already recognised that NIR spectroscopy originates from overtones and combinations of fundamentals. In the 1950s, NIR spectroscopy received considerable interest in the hydrogen bonding and anharmonicity studies. , $^{71,22}$ However, the development of NIR spectroscopy was more delayed compared to that of MIR spectroscopy, owing to the insufficient development in the spectrometers and difficulty in spectral analysis.

It was not a spectroscopist, but an agricultural engineer, Karl Norris of the United States Department of Agriculture, who paved the way for the innovative development of NIR spectroscopy. ${ }^{5-7}$ He and his colleagues attempted to utilise NIR spectroscopy for the quality assessment of agricultural products. Norris proposed the application of statistical methods to build calibration models from NIR data. His idea led to the realisation of the advantages of NIR spectroscopy in practical applications. Camille Sandorfy was a pioneer in the basic studies of NIR spectroscopy. He is particularly famous for his research on the relationship between anharmonicity and hydrogen bonds. ${ }^{7,21}$

As for FIR spectroscopy, its history is unclear; however, it is evident that this technique was already used actively in the 1960s. ${ }^{8,9}$ In 1970 and 1971, two famous FIR books were published. ${ }^{8,9}$ In these books, one can find many interesting papers on FIR published in the 1960s and 1950s. In contrast to MIR spectroscopy, the development of FIR spectroscopy was behind, probably because the development of the instruments and equipments that were compatible in the FIR region was rather slow. Further, the introduction of the FT technique to the FIR region was unsuccessful in the 1970s.

$\mathrm{THz}$ spectroscopy emerged in the 1990s. ${ }^{10-12}$ Advances in optics and electronics in the 1990s resulted in the development of various types of $\mathrm{THz}$ sources and sensors. $\mathrm{THz}$ sources include free electron lasers, synchronous, narrow-band quantum cascade lasers, and broadband generation from ultrafast pulsed lasers. The most common broadband $\mathrm{THz}$ sources are the benchtop pulsed Ti:sapphire lasers. Nowadays, $\mathrm{THz}$ timedomain spectroscopy (THz-TDS) is commonly used for applications in chemistry. ${ }^{10-12}$ Further, photoconductive antenna type and electro-optics (EO) type detectors are used.

\section{MIR Spectroscopy}

\subsection{Principles and characteristics of MIR spectroscopy 2.1.1 Principles of MIR spectroscopy}

In this section, I briefly explain the principles of MIR spectroscopy. ${ }^{2-4}$ For more details one can learn them in many text books. ${ }^{2-4}$ The principles of MIR spectroscopy are also those of NIR and FIR or THz spectroscopy.

At room temperature, most of the molecules exist in the ground vibrational state. Thus, a transition from state $v^{\prime \prime}=0$ to $v^{\prime \prime}=1$ (first excited state) is possible. The absorption that corresponds to this transition is called fundamental. Although most bands that are observed in the IR absorption spectrum are due to the fundamental absorption, in some cases, it is possible to observe bands that correspond to the transitions from state $v^{\prime \prime}=0$ to $v^{\prime \prime}=2$. They are called the first overtones. For example, the fundamentals of $\mathrm{C}=\mathrm{O}$ stretching bands appear in the $1750-1700 \mathrm{~cm}^{-1}$ region, and their first overtones appear in the $3450-3350 \mathrm{~cm}^{-1}$ region. Bands that correspond to the transitions from state $v^{\prime \prime}=0$ to $v^{\prime \prime}=2,3$, or 4 may be observed in the NIR spectra. Bands due to combinations are observed in the MIR and NIR spectra.

\subsubsection{Characteristics and advantages of MIR spectroscopy}

The characteristics and advantages of MIR spectroscopy are summarised as follows. ${ }^{2-4}$

1 MIR spectroscopy is a highly sensitive method. Many people do not always recognise this important point but note that the IR spectrum can be measured even from a monolayer.

2 MIR spectroscopy handles functional groups. Polar functional groups, such as $\mathrm{C}=\mathrm{O}, \mathrm{OH}$, and $\mathrm{C}-\mathrm{S}$ groups, exhibit intense bands.

3 Local modes, such as the $\mathrm{CH}_{2}$ scissoring mode, give rise to strong bands in an IR spectrum, while vibrational modes due to a large part of a molecule or an entire molecule, such as an accordion mode, yield very weak or no bands.

4 Bands, arising from antisymmetric stretching modes, are stronger than those derived from symmetric stretching modes.

5 Among the MIR bands shown by a benzene ring, the bands due to ring stretching modes in the $1600-1450 \mathrm{~cm}^{-1}$ region and those derived from $\mathrm{CH}$ out-of-deformation modes in the $900-700 \mathrm{~cm}^{-1}$ region are strong.

6 Apart from the MIR absorption spectroscopy, one can also use MIR reflection, attenuated total reflection (ATR), emission, PAS, and VCD.

7 The connection of MIR spectrometers with other instruments, such as microscope, gas chromatograph, and liquid chromatograph, is relatively easy. It is also possible to combine an MIR spectrometer with an atomic force microscopy instrument. Various types of hyphenated technologies, including MIR spectroscopy, are available.

\subsection{Applications of MIR spectroscopy}

The applications of MIR spectroscopy are wide; however, we may divide them into two major fields: applications in analytical chemistry and those in physical chemistry. ${ }^{2-4}$ Of course, many applications lie somewhat in between these two fields. The analytical applications are summarised as follows:

1 Identification of molecules: MIR spectra are called as the fingerprints of molecules.

2 Qualitative analysis, discriminant analysis, and differentiation. ${ }^{23}$

3 Quantitative analysis.

4 Trace analysis and minute analysis via micro MIR.

5 Nanoscale spatial resolution analysis via nanoIR. ${ }^{16}$

6 Micro distribution analysis via MIR imaging. ${ }^{17,18}$

Additionally, the MIR spectroscopy can be used for numerous applications in physical chemistry, ${ }^{2-4}$ such as

1 Molecular structure, molecular interactions, hydrogen bonds, conformation, and orientation.

2 Structure of molecular assemblies, such as thin films, selfassembled monolayers, and biological membranes; molecular packing.

3 Chemical reactions and molecular dynamics. One can use time-resolved MIR ${ }^{2,3 a}$ and $2 \mathrm{D}^{-\mathrm{IR}^{15}}$ for exploring the molecular dynamics, excited states, and reaction intermediates.

4 Molecular orientation studies and surface analysis. ${ }^{4}$ Various experimental and spectral analysis methods, such as ATR, RA, external reflection, specular reflection, and MAIRS ${ }^{20}$ have been used.

In this review, I have introduced three examples of the applications of MIR spectroscopy. 

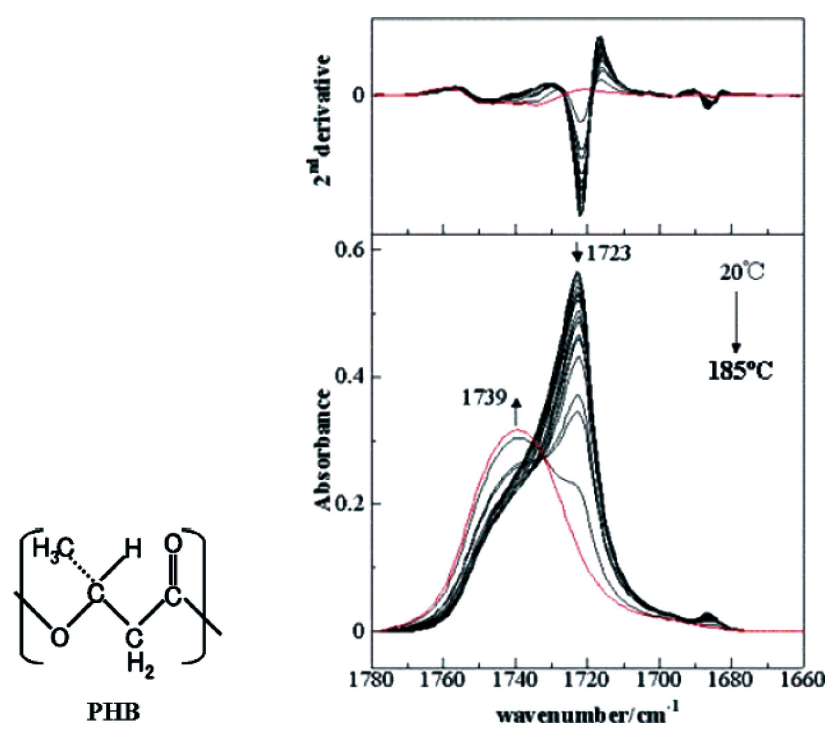

Fig. 2 Chemical structure of poly(3-hydroxybutylate) (PHB) and temperature-dependent MIR spectra in the $\mathrm{C}=\mathrm{O}$ stretching band region of PHB in a film measured in a temperature range of $20-185^{\circ} \mathrm{C}$ (Reproduced from Ref. 27b with permission).

\subsubsection{Investigation of hydrogen bonding via MIR spectro- scopy}

MIR spectroscopy has a long history in hydrogen bonding research. ${ }^{2,3,24-28}$ Bands due to $\mathrm{OH}, \mathrm{NH}$, and $\mathrm{C}=\mathrm{O}$ stretching modes are strong in the MIR spectra, and shift sensitively depending on the strength of the hydrogen bond. Recently, it has been shown that MIR spectroscopy is also useful for investigating weak hydrogen bonds. ${ }^{25-28}$

Sato et al..$^{27 b, 28}$ conducted studies on weak hydrogen bonds of various types of biodegradable polymers using MIR spectroscopy. They investigated a $\mathrm{CH} \cdots \mathrm{O}=\mathrm{C}$ hydrogen bond in poly(3-hydroxybutylate) (PHB) (Fig. 2). Figure 2 shows the temperature-dependent MIR spectra in the $\mathrm{C}=\mathrm{O}$ stretching band region of $\mathrm{PHB}$ in a film measured over a temperature range of $20-185^{\circ} \mathrm{C} .{ }^{27 \mathrm{~b}}$ The second derivative spectra are also shown in Fig. 2. Two bands are observed in the $\mathrm{C}=\mathrm{O}$ stretching region. Sato et al. ${ }^{27 \mathrm{~b}}$ assigned the bands at 1723 and $1740 \mathrm{~cm}^{-1}$ to the $\mathrm{C}=\mathrm{O}$ stretching mode of the crystalline and amorphous parts of $\mathrm{PHB}$, respectively. In the MIR spectra of esters, the $\mathrm{C}=\mathrm{O}$ stretching band appears near 1740 and $1700 \mathrm{~cm}^{-1}$, for the free $\mathrm{C}=\mathrm{O}$ and hydrogen-bonded $\mathrm{C}=\mathrm{O}$ groups, respectively. Thus, Sato et al. ${ }^{27 \mathrm{~b}}$ ascribed the $1723 \mathrm{~cm}^{-1}$ band to the $\mathrm{C}=\mathrm{O}$ stretching mode of a weak $\mathrm{CH} \cdot \mathrm{\cdots} \mathrm{C}=\mathrm{O}$ hydrogen bond of $\mathrm{PHB}$ based together on other evidences described later.

Figures 3(a) and 3(b) show temperature-dependent MIR spectra and their second derivatives in the $3050-2850 \mathrm{~cm}^{-1}$ region of $\mathrm{PHB}$ in a film measured over a temperature range of $20-185^{\circ} \mathrm{C} .{ }^{27 b}$ In this region, several bands due to $\mathrm{CH}_{3}$ and $\mathrm{CH}_{2}$ stretching modes are expected to appear. Usually, these bands appear below $3000 \mathrm{~cm}^{-1}$. However, note that a shoulder appears at approximately $3009 \mathrm{~cm}^{-1}$ (Fig. 3). This shoulder can be clearly detected in the second derivative spectra. Interestingly, this band shifts from 3009 to $3004 \mathrm{~cm}^{-1}$ as the temperature increases from 20 to $180^{\circ} \mathrm{C}$, as can be in the second derivative spectra. ${ }^{27 \mathrm{~b}}$ It is well known that the $\mathrm{CH}_{3}$ stretching band appears above $3000 \mathrm{~cm}^{-1}$ when a $\mathrm{CH}_{3}$ group forms a weak hydrogen bond with an $\mathrm{O}$ atom ( $\mathrm{CH}$... $\mathrm{O}$ hydrogen bond). ${ }^{29}$ Therefore, Sato et al. ${ }^{27 \mathrm{~b}}$ interpreted the emergence of the $\mathrm{CH}_{3}$ band at $3009 \mathrm{~cm}^{-1}$ as an evidence proving the existence of weak

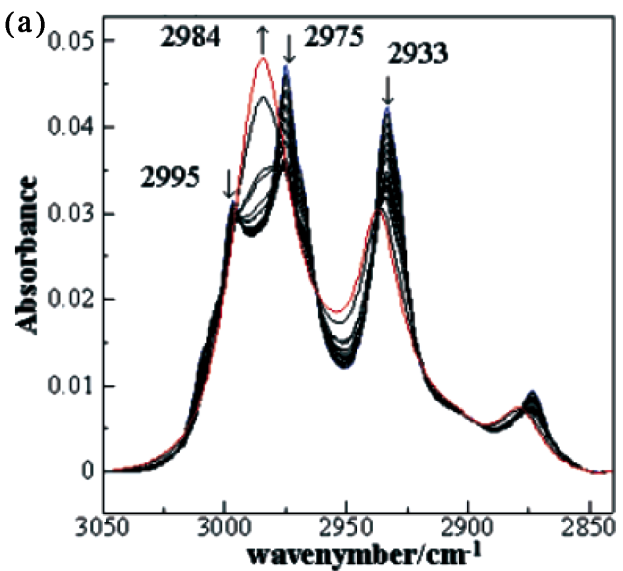

(b)

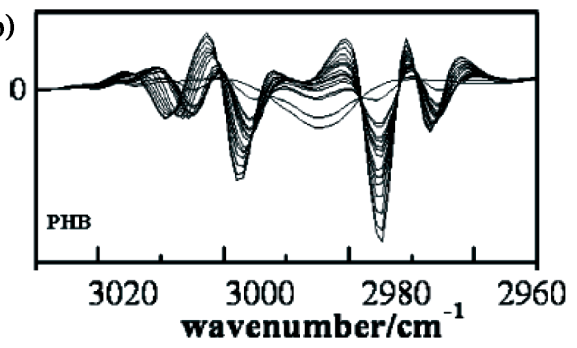

Fig. 3 (a) Temperature-dependent MIR spectra and (b) their second derivatives in the region of $3050-2850 \mathrm{~cm}^{-1}$ of PHB in a film collected over a temperature range of 20 to $185^{\circ} \mathrm{C}$ (Reproduced from Ref. $27 \mathrm{~b}$ with permission).

\section{$\mathrm{CH}$... $\mathrm{O}=\mathrm{C}$ hydrogen bond in $\mathrm{PHB}$.}

Based on the evidences obtained by MIR spectroscopy, wideangle x-ray diffraction (WAXD), and the calculation of van der Waals radii, Sato et al. ${ }^{27 \mathrm{~b}}$ concluded that an intermolecular hydrogen bond, $\mathrm{C}-\mathrm{H} \cdots \mathrm{O}=\mathrm{C}$, between one of the $-\mathrm{C}-\mathrm{H}$ groups in the $\mathrm{CH}_{3}$ group in one helix and the $-\mathrm{C}=\mathrm{O}$ group in the other helix is formed along the $a$-axis, and that the hydrogen bond stabilises the chain folding in PHB lamellar crystallites. Recently, Wang et al. confirmed the existence of $\mathrm{C}-\mathrm{H} \cdots \mathrm{O}=\mathrm{C}$ hydrogen bond in PHB using high-resolution X-ray crystallography. ${ }^{30}$ Sato et al. also investigated the $\mathrm{CH} \cdot \cdots \mathrm{O}=\mathrm{C}$ hydrogen bond in PHB using $\mathrm{NIR}^{27 \mathrm{c}}$ and FIR or $\mathrm{THz}^{27 \mathrm{~d}}$ spectroscopy.

2.2.2 Anharmonic DFT study of aliphatic ethers; influence of non-fundamental modes on MIR spectra

Quantum chemical calculations of MIR spectra have become a routine technique in the last few decades. ${ }^{31,32}$ Harmonic approximation provides a reasonably accurate prediction of the bands observed in the MIR spectra. To improve the agreement between the positions of the measured fundamental bands and calculated harmonic frequencies, the idea of scaling factors has been introduced. ${ }^{33}$ While harmonic calculations with scaling factors form a formidable tool of practical value for MIR spectroscopy, this approach has apparent intrinsic limitations. Recently, anharmonic calculations have been developed significantly for MIR and NIR spectroscopy. ${ }^{32,34,35}$

Bec et al. ${ }^{34 a}$ calculated the fundamental and non-fundamental vibrational modes, first overtone, and binary combination modes of selected aliphatic ethers using a fully anharmonic generalised second-order vibrational perturbation theory (GVPT2) on the DFT-B2PLYP/SNST level. They considered the conformational isomers of the studied molecules for modelling the line shapes theoretically. A comparison between the calculated and 
experimental spectra of the aliphatic ethers revealed that the MIR spectra of ethers are influenced by the bands arising from the non-fundamental modes, particularly from the combination modes in the C-H stretching region $\left(3200-2800 \mathrm{~cm}^{-1}\right)$. $^{34 \mathrm{a}}$ Owing to the effect of vibrational resonances, the intensities of the overtones and combination bands due to $\mathrm{CH}_{3}$ and $\mathrm{CH}_{2}$ deformation modes increase sufficiently to affect the spectral envelope in this region. The anharmonic DFT calculations reproduced the line shapes in the $2700-1600 \mathrm{~cm}^{-1}$ region, where only the bands originating from the non-fundamental vibrations appear. Thus, it is concluded that this anharmonic approach enables accurate reproduction of complex MIR line shapes. ${ }^{34 \mathrm{a}}$

Bec et al. ${ }^{34 \mathrm{a}}$ compared the theoretical and experimental spectra of seven aliphatic ethers (di- $n$-propyl ether, DNPE; di-iso-propyl ether, DIPE; $n$-butyl methyl ether, NBME; $n$-butyl ethyl ether, NBEE; di- $n$-butyl ether, DNBE; tert-butyl methyl ether, TBME; and tert-amyl methyl ether, TAME). The theoretical spectra were obtained by applying the GVPT2 approach to the DFTB2PLYP/SNST level of theory. ${ }^{34 a}$ They focused on investigating how the overtones and combination bands influence the experimental spectral envelopes of these ethers. They especially focused on Fermi resonances, through which the influence of non-fundamental bands increased considerably.

Figure 4 compares the experimental and calculated MIR spectra in the $\mathrm{C}-\mathrm{H}$ stretching region of DNBE. ${ }^{34 a}$ The calculated spectrum contains contributions from 10 rotational isomers. It reproduces the experimental spectrum well. Bands due to the combination modes involving $\mathrm{CH}_{2}$ deformation modes, mainly scissoring and wagging modes, and asymmetric deformation modes of $\mathrm{CH}_{3}$ groups are noted in this region. Bands derived from the overtones of $\mathrm{CH}_{2}$ scissoring and $\mathrm{CH}_{3}$ asymmetric deformation can also be identified in the $3000-2940 \mathrm{~cm}^{-1}$ region, although the influence is less pronounced. These nonfundamentals contribute largely to the envelope of the experimental spectrum, particularly in the $2980-2880 \mathrm{~cm}^{-1}$ region. The proposed band assignments for major contributions from non-fundamentals based on PED analysis are found in Ref. 34a (B2PLYP/SNST). ${ }^{34 a}$

Figure 5A depicts the experimental and calculated IR spectra of DNBE in the $2700-1500 \mathrm{~cm}^{-1}$ region. ${ }^{34 a}$ In this region, all the observed bands are assigned to combinations or overtones. Notably, the calculated spectrum accurately reproduces the experimental spectrum. The relatively intense bands at 2404 ,
2086, and $1953 \mathrm{~cm}^{-1}$ are assigned to $2404 \mathrm{~cm}^{-1}$ (major contributions: $\quad v \mathrm{CO}+\delta_{\text {twist }} \mathrm{CH}_{2}, \quad \delta_{\text {twist }} \mathrm{CH}_{2}+\delta_{\text {as }} \mathrm{CH}_{3}, \quad v \mathrm{CC}+$ $\delta_{\text {wagg }} \mathrm{CH}_{2}, \quad v \mathrm{CO}+\delta_{\text {wagg }} \mathrm{CH}_{2}$ ), $2086 \mathrm{~cm}^{-1}$ (major contributions: $\left.v \mathrm{CC}+v \mathrm{CO}, \delta_{\text {rock }} \mathrm{CH}_{3}+v \mathrm{CO}, \delta_{\text {rock }} \mathrm{CH}_{2}+v \mathrm{CO}\right)$, and $1953 \mathrm{~cm}^{-1}$ (mainly $v \mathrm{CC}+v \mathrm{CO}, \delta_{\text {rock }} \mathrm{CH}_{2}+v \mathrm{CO}, \delta \mathrm{CCC}+\delta_{\text {sciss }} \mathrm{CH}_{2}$ ). The higher absorbance of these bands originates from the involvement of the intense $\mathrm{C}-\mathrm{O}$ stretching mode, which has a relatively large transition dipole moment.

Figure 5B compares the experimental and calculated MIR spectra in the $1550-600 \mathrm{~cm}^{-1}$ region of DNBE. ${ }^{34 a}$ In this region, the influence of anharmonicity is noted in the $\mathrm{C}-\mathrm{O}$ stretching region $\left(1180-1080 \mathrm{~cm}^{-1}\right)$, and almost all non-

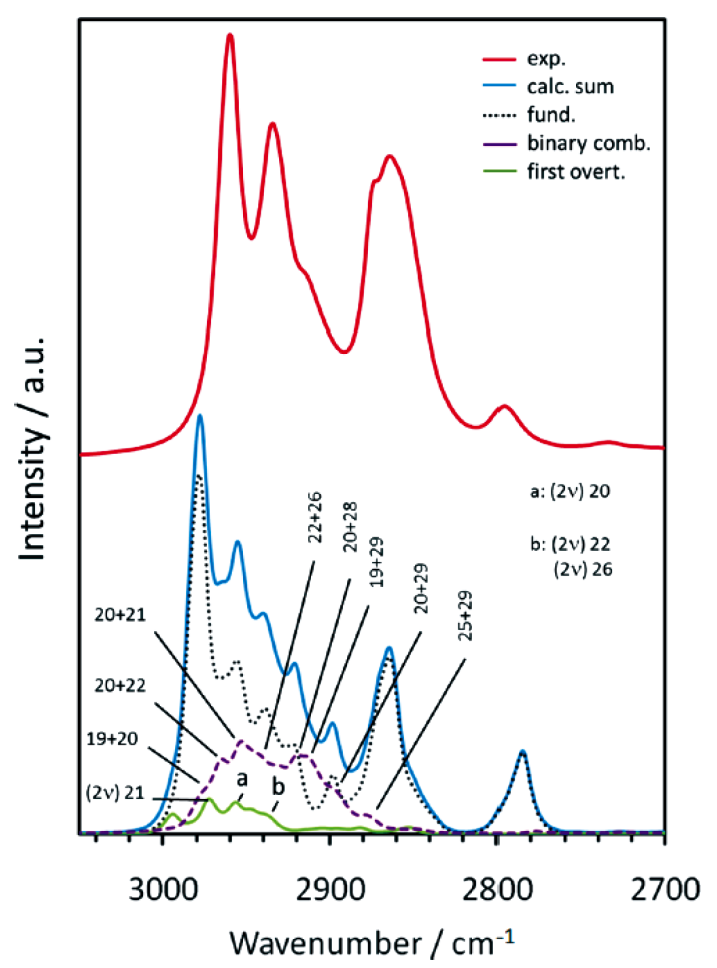

Fig. 4 Comparison of experimental and calculated IR spectra in the C-H stretching region (3050 - $2700 \mathrm{~cm}^{-1}$ ) of DNBE (Reproduced from Ref. 34a with permission).
A.

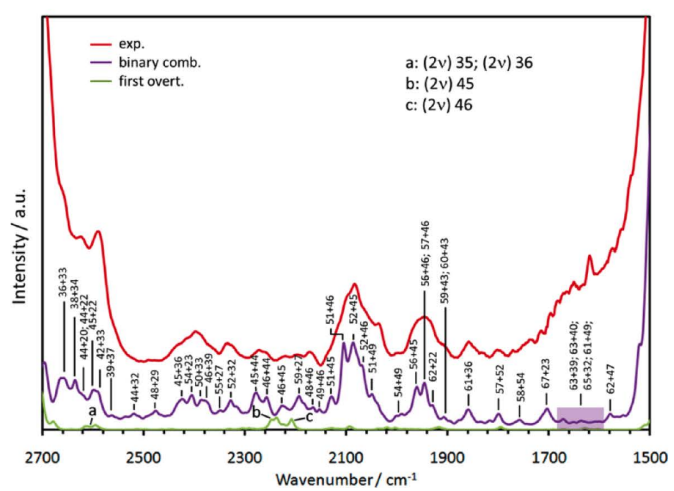

B.

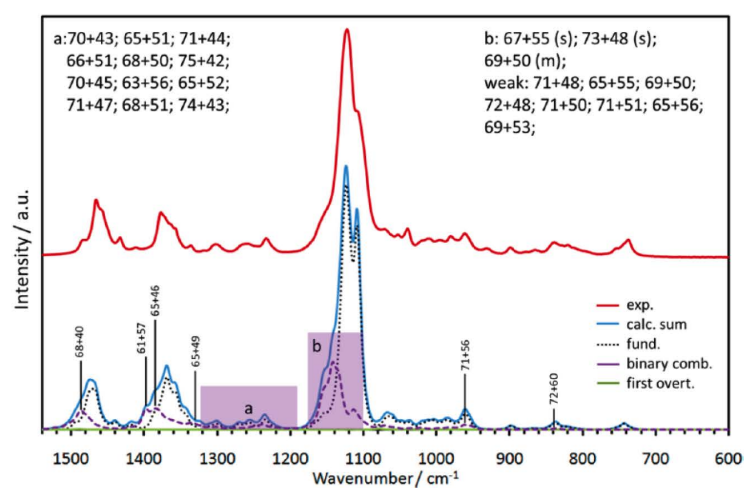

Fig. 5 A. Comparison of experimental and calculated IR spectra in the $2700-1500 \mathrm{~cm}^{-1}$ region of DNBE. B. Comparison of experimental and calculated IR spectra in the $1550-600 \mathrm{~cm}^{-1}$ region of DNBE (Reproduced from Ref. 34a with permission). 


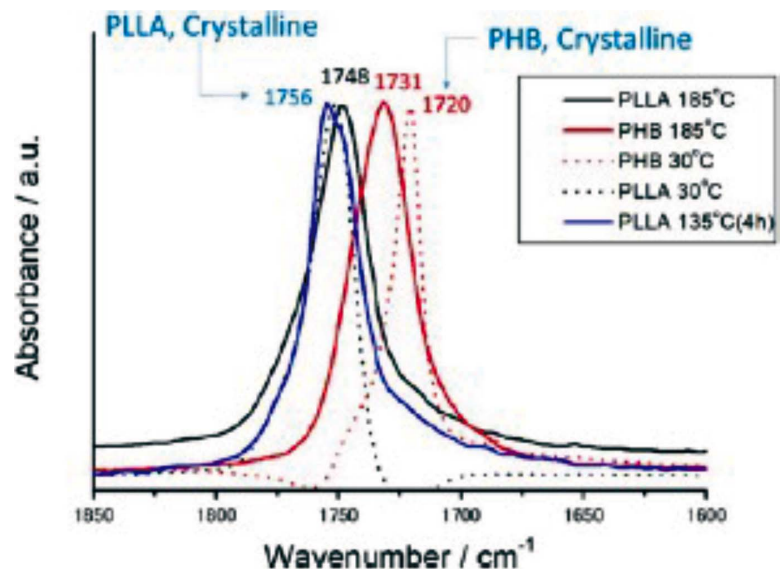

Fig. 6 ATR-FTMIR spectra in the $\mathrm{C}=\mathrm{O}$ stretching band region of PHB and PLLA homopolymers at the molten state $\left(185^{\circ} \mathrm{C}\right)$, isothermal crystallization temperature $\left(135^{\circ} \mathrm{C}\right.$ ), and $30^{\circ} \mathrm{C}$ (Reproduced from Ref. 37 with permission).

fundamentals are combination modes.

Bec et al. ${ }^{34 \mathrm{~b}}$ also conducted another anharmonic calculation of the MIR spectra. They combined the periodic harmonic calculations with the anharmonic corrections based on finite models for the MIR spectra of crystalline nucleobases.

2.2.3 In situ ATR-FTMIR spectroscopic imaging for simultaneous visualisation of phase separation and crystallisation in PHB/poly(L-lactic acid) (PLLA) blends

Nowadays, ATR-FTMIR imaging is a crucial technique in analytical as well as physical chemistry. ${ }^{17,18,36}$ One can use this technique for visualising the distributions of components and for observing physical properties, such as crystallinity. ATRFTMIR allows us to study the mechanism of phase transition and crystallisation in various materials in situ. This technique has also been applied to analyse various biomedical problems. ${ }^{17,18,36}$

Sato et al. ${ }^{37}$ applied the ATR-FTIR spectroscopic imaging technique to visualise the phase separation and crystallisation of $\mathrm{PHB} /$ poly(L-lactic acid) (PLLA) blends in films simultaneously. From the peak shift of the $v(\mathrm{C}=\mathrm{O})$ band of PHB during the isothermal crystallisation process, it was found that a transformation of the intermolecular interaction between PHB and PLLA occurred in the miscible state to the inter- and intramolecular interactions between the PHBs in the immiscible state. The obtained ATR-FTMIR spectroscopic images showed the appearance and gradual separation of the crystalline PHBrich domains and crystalline PLLA-rich domains, thereby clearly illustrating the entire dynamical process of phase separation.

Figure 6 shows the ATR-FTMIR spectra of PHB and PLLA homopolymers in the $\mathrm{C}=\mathrm{O}$ stretching band region at their molten state $\left(185^{\circ} \mathrm{C}\right)$, isothermal crystallisation temperature $\left(135^{\circ} \mathrm{C}\right)$, and $30^{\circ} \mathrm{C}^{37}$ The bands at 1731 and $1748 \mathrm{~cm}^{-1}$ at $185^{\circ} \mathrm{C}$ are assigned to the $\mathrm{C}=\mathrm{O}$ stretching $(v(\mathrm{C}=\mathrm{O}))$ modes of the amorphous states of PHB and PLLA, respectively. The band at $1720 \mathrm{~cm}^{-1}$ is due to the $v(\mathrm{C}=\mathrm{O})$ mode of crystalline $\mathrm{PHB}$, as the intensity of this band increases during the isothermal crystallisation of pure PHB, while that at $1740 \mathrm{~cm}^{-1}$ of amorphous PHB decreases. Similarly, a band at $1756 \mathrm{~cm}^{-1}$ originates from the $v(\mathrm{C}=\mathrm{O})$ mode of crystalline PLLA because the intensity of this band increases during the isothermal crystallisation of pure PLLA, while the intensity of the band at $1748 \mathrm{~cm}^{-1}$, which arises due to amorphous PLLA, decreases.

Figure 7 displays the ATR-FTMIR spectroscopic images of
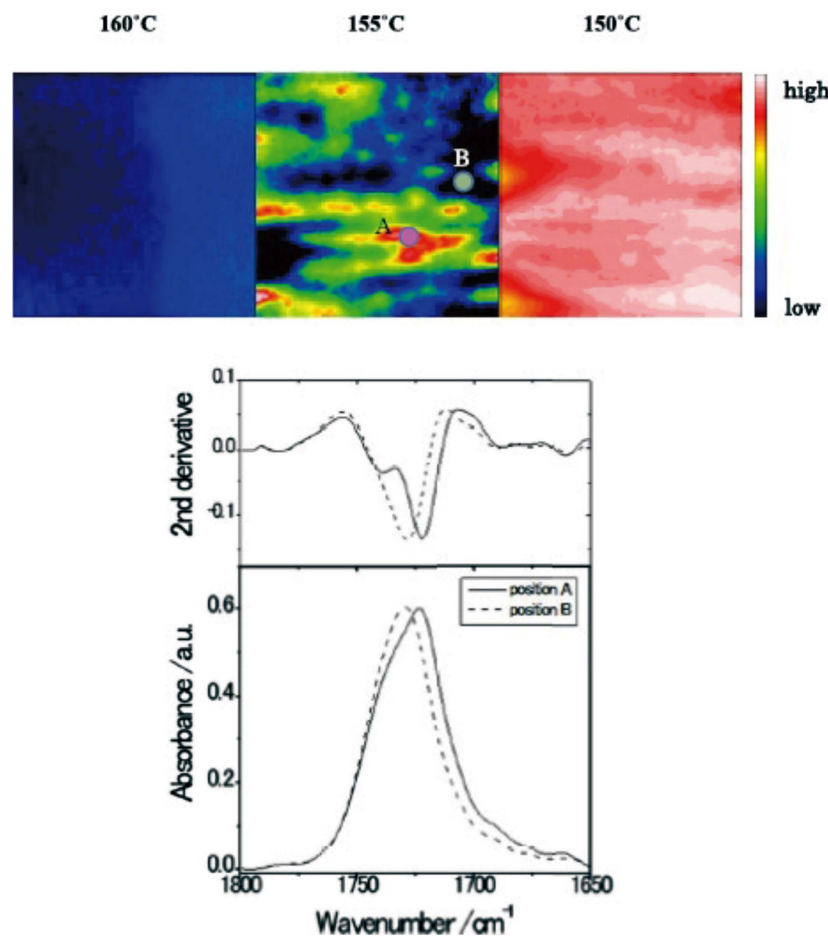

Fig. 7 (top) The ATR-FTIR spectroscopic images of PHB homopolymer after annealing at 160,155 , and $150^{\circ} \mathrm{C}$, separately (Reproduced from Ref. 37 with permission). (bottom) MIR spectra and their second derivatives measured at Point A and B.

the PHB homopolymer after annealing at 160,155 , and $150^{\circ} \mathrm{C}$, separately. ${ }^{37}$ These images are constructed based on the integrated absorbance of the $v(\mathrm{C}=\mathrm{O})$ band of crystalline $\mathrm{PHB}$ $\left(1730-1700 \mathrm{~cm}^{-1}\right)$. Figure 7 reveals that, when the isothermal crystallisation temperature of $\mathrm{PHB}\left(160^{\circ} \mathrm{C}\right)$ is close to its melting temperature $\left(163^{\circ} \mathrm{C}\right)$, PHB does not crystallise, even after $6 \mathrm{~h}$. At $155^{\circ} \mathrm{C}$, small PHB crystals are observed in the imaged area; however, amorphous PHB remains partially crystallised. MIR spectra and their second derivatives shown at the bottom of Fig. 7 indicate that, at Point A, PHB is partially crystalised, whereas at Point $\mathrm{B}$, it is in an amorphous state. ${ }^{37}$ When the isothermal crystallisation temperature decreases to $150^{\circ} \mathrm{C}$ or below, crystalline PHB dominates the entire imaged area. Hence, Sato et al. ${ }^{37}$ concluded that the critical crystallisation temperature of the molten state $(\mathrm{Tc})$ lies between 155 and $160^{\circ} \mathrm{C}$ for PHB. Conversely, the crystalline PLLA occupies the entire imaged area over the entire isothermal crystallisation temperature range $\left(130-160^{\circ} \mathrm{C}\right)$. As the isothermal crystallisation temperature $\left(160^{\circ} \mathrm{C}\right)$ is far from the melting temperature $\left(182^{\circ} \mathrm{C}\right)$ of PLLA, it can be concluded that the Tc of PLLA is above $160^{\circ} \mathrm{C}$.

Figure 8 (top and middle) shows the ATR-FTMIR spectroscopic images of the PHB/PLLA blend acquired immediately after the temperature decreases to $150^{\circ} \mathrm{C}$, after 60 , 120, 180, and 360 min..$^{37}$ Notably, in Fig. 8, both PHB crystalrich domains and PLLA crystal-rich domains emerge and separate gradually, indicating that phase separation takes place during the isothermal crystallisation. The ATR-FTMIR spectroscopic image of the PHB/PLLA blend, measured $60 \mathrm{~min}$ after the temperature reached $150^{\circ} \mathrm{C}$, shows that no PHB crystal was found in the imaged area. This suggests that PHB has not yet started crystallising. In contrast, PLLA has already started crystallising at that time, and PLLA crystals appear with a high 

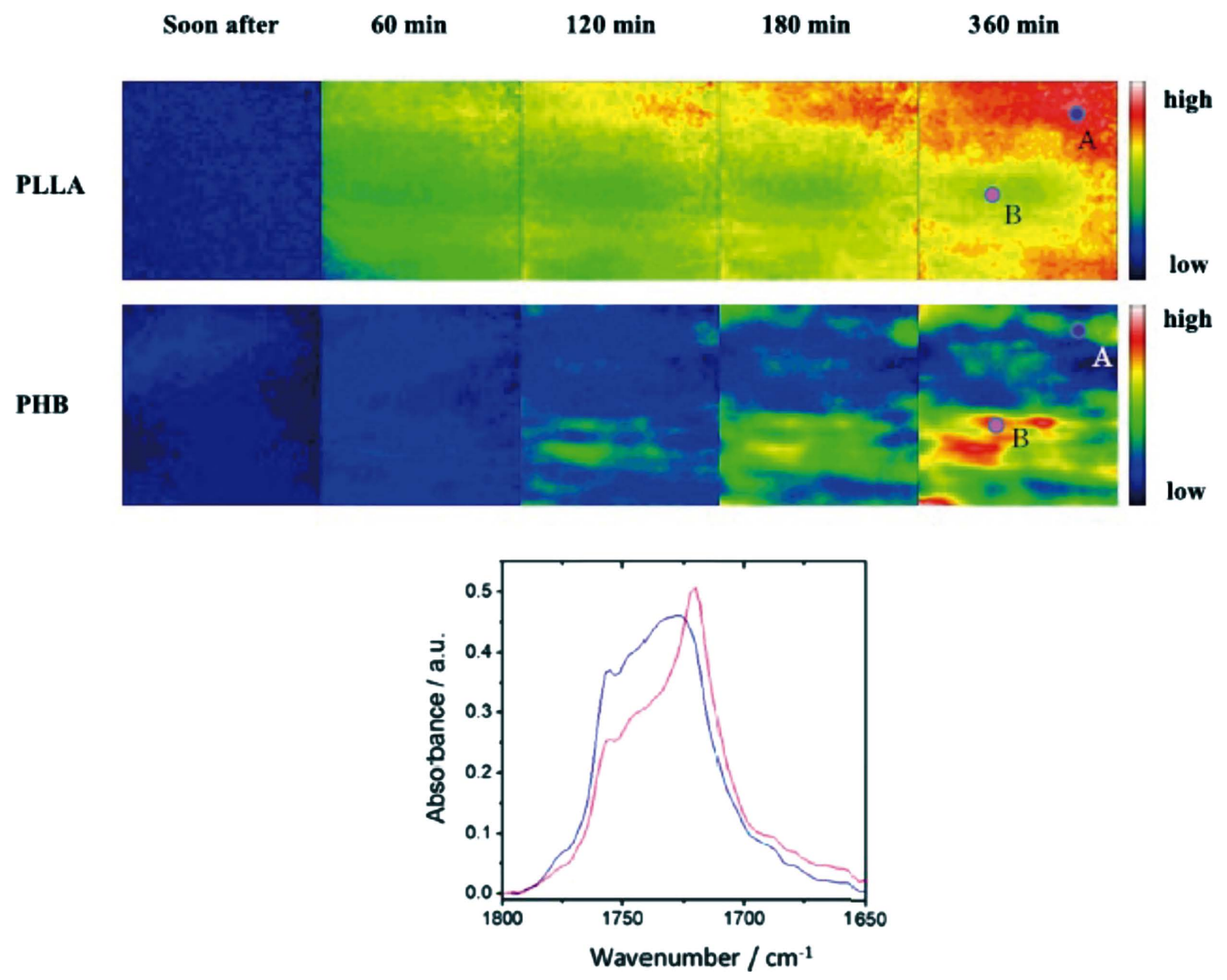

Fig. 8 (top and middle) The ATR-FTIR spectroscopic images of the PHB/PLLA blend acquired immediately after the temperature decrease to $150^{\circ} \mathrm{C}, 60,120,180$, and 360 min later. (bottom) MIR spectra measured at Point A (blue) and B (purple) (Reproduced from Ref. 37 with permission).

concentration at the top of the imaged area; however, their concentration is relatively low at the middle and bottom left sides of the image. Thus, it is very likely that the phase separation of the polymer blend begins before the initiation of the crystallisation process.

As shown in Fig. 8 (bottom), the spectra were extracted from the PLLA crystal-rich domain, indicated by a dark blue circle (position A) on the top-right side, and the PHB crystal-rich domain, which is depicted by a pink circle (position B) on the middle-right side. ${ }^{37}$ Notably, the concentration of PLLA crystals in the PLLA crystal-rich domains is higher than that in the PLLA crystal-poor domains. Similarly, the concentration of PHB crystals in the PHB crystal-rich domains is higher than that in the PHB crystal-poor domains. As the isothermal crystallisation time increases, the amorphous polymer transforms into a crystalline polymer, and the extent of phase separation in the polymer blend is enhanced.

This study demonstrated that in situ ATR-FTIR spectroscopic imaging is a powerful technique for simultaneously visualising the crystallisation and phase separation of the PHB/PLLA blend. ${ }^{37}$ We can also investigate the underlying mechanism of the phase separation and crystallisation in the polymer blend via the imaging results.

\section{NIR Spectroscopy}

3.1 Principles and characteristics of NIR spectroscopy 3·1·1 Principles of NIR spectroscopy

One of the most characteristic features of NIR spectroscopy is the weak or very weak bands that occur in the NIR region..$^{5-7}$ Both bands, the ones originating from electronic transitions $s^{6,7,38}$ and those arising from overtones and combination modes, are weak. The overtones and combination modes are the so-called forbidden transitions. The fact that the bands in the NIR region are weak or very weak is what makes this region unique and markedly different from the other regions. The NIR region is valuable because this is the sole region that serves as a high transmission window to radiation.

3.1.2 Characteristics and advantages of NIR spectroscopy

We can summarise the characteristics of NIR bands as follows..$^{5-7}$

1) NIR bands are much weaker than MIR bands. The NIR bands become weaker as the wavelength decreases because bands due to higher-order overtones and ternary combinations appear in the shorter wavelength region.

2) Bands due to overtones and combination modes overlap with each other. A NIR spectrum often shows multicollinearity. To solve this chemometrics is used. Additionally, numerous bands, arising from the Fermi resonance, emerge in the NIR region; thus, the assignment of NIR bands is generally not easy.

3) Most of the bands in the NIR region are attributed to functional groups containing a hydrogen atom $(e . g . \mathrm{OH}$, $\mathrm{CH}, \mathrm{NH}$ ). This is partly because the anharmonic constant of an $\mathrm{XH}$ bond is large, and partly because a fundamental XH stretching vibration has a high frequency (3800 $\left.2800 \mathrm{~cm}^{-1}\right)$. Thus, NIR spectroscopy can also be called "an XH spectroscopic method".

4) For the XH bands, the first overtone band shifts, as in the 

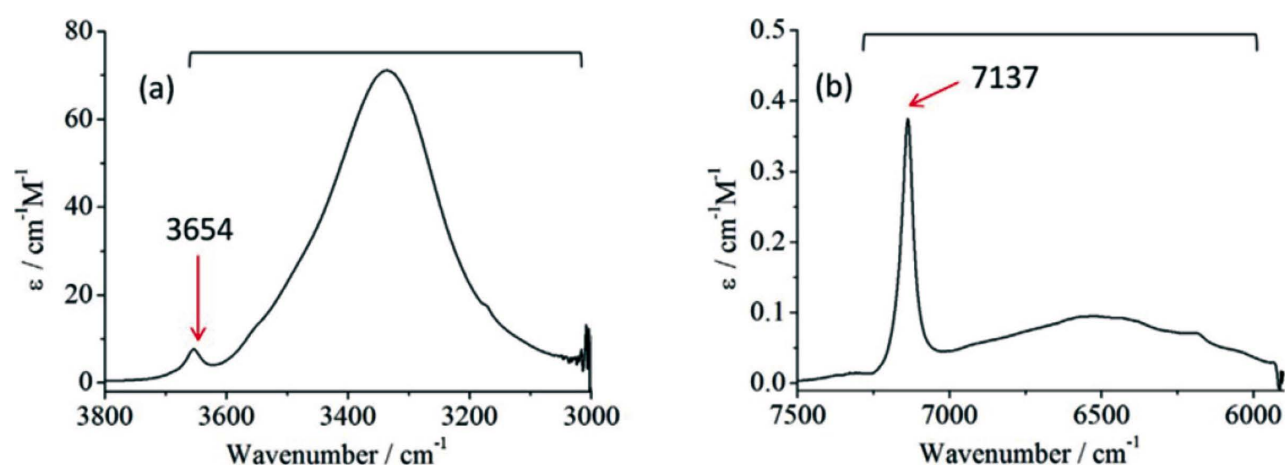

Fig. 9 Comparison of a MIR spectrum of $0.5 \mathrm{M}$ methanol- $d_{3}$ in $n$-hexane with its corresponding NIR spectrum (Reproduced from Ref. 40 with permission).

case of an MIR spectrum, owing to the formation of a hydrogen bond and an interaction between the molecules. The shift is approximately double for an NIR band than that for the corresponding MIR band.

5) In the NIR region, the $\mathrm{OH}$ and $\mathrm{NH}$ stretching bands of monomeric and polymeric species are better separated as compared to those in the MIR region. Additionally, in the NIR region, one can differentiate between the bands that are ascribed to the terminal free $\mathrm{OH}$ or $\mathrm{NH}$ groups of the polymeric species from those ascribed to their free $\mathrm{OH}$ or $\mathrm{NH}$ groups.

6) Owing to the larger anharmonicity, the first overtone bands of $\mathrm{OH}$ and $\mathrm{NH}$ stretching modes of monomeric species are much more enhanced as compared to those arising from polymeric species.

Almost all of the above characteristics are derived from the fact that NIR spectroscopy incorporates forbidden transitions within the harmonic oscillator approximation.

One can discuss the advantages of NIR spectroscopy in comparison with those of MIR spectroscopy as follows. ${ }^{5-7}$

1) NIR spectroscopy enables in situ analysis with a sample in its original form. Although one can utilise ATR or PAS for in situ MIR spectroscopic analysis, there is no choice other than NIR spectroscopy if one wishes to measure an absorption spectrum of an entire apple or a human head. NIR spectroscopy is also suitable for non-destructive or non-invasive analysis of thick samples.

2) In general, NIR spectroscopy is more suitable for the analysis of aqueous solutions than MIR spectroscopy because the intensity of water bands is much weaker in the NIR spectrum than in the MIR spectrum.

3) In NIR spectroscopy, a fibre optic probe can be set in a dangerous environment and remotely manipulated. This is one of the reasons why NIR spectroscopy is appropriate for on-line analysis. Light fibres operating in the MIR region are expensive and inefficient.

4) In NIR spectroscopy, one can select a light path length freely. In contrast, MIR spectroscopy usually requires a cell with a very short path length. NIR spectroscopy allows one to use a $0.1 \mathrm{~mm}$ cell, a $1 \mathrm{~cm}$ cell, or even a $10 \mathrm{~cm}$ cell

5) Optical materials that are used in the NIR region are cheaper than those used in the MIR region; for instance, we can use glass cells.

\subsection{Applications of NIR spectroscopy}

The applications of NIR spectroscopy are diverse; however, as in the case of MIR spectroscopy, they may be divided into two major fields: applications in analytical chemistry and physical chemistry. The applications of NIR spectroscopy are summarised as follows. ${ }^{5-7}$

1) In situ, non-destructive, and non-invasive analysis; analysis of bulk materials.

2) Qualitative analysis, discriminant analysis, and differentiation.

3) Quantitative analysis.

4) On-line monitoring and analysis using light fibres.

5) Visualisation of the distribution of components and physical properties by NIR imaging; monitoring of water and organic solvent dispersion by NIR imaging.

6) Quality control, such as process analysis technology.

One can use NIR spectroscopy for numerous applications in physical chemistry, such as:

1) Studies on the molecular interactions, hydrogen bonds, hydration, phase separation, and miscibility.

2) Molecular orientation of thick samples.

3) Chemical reactions, molecular dynamics, including industrial applications.

4) Studies of physical properties, such as crystallinity of thick samples and bulk materials.

5) Solution chemistry.

\subsubsection{Studies on hydrogen bonding via NIR spectroscopy}

NIR spectroscopy has long been used for hydrogen bonding studies. ${ }^{5-7,21,22,38,39}$ In the 1950 s, NIR spectroscopy was already noted to be useful for hydrogen bond investigations. ${ }^{21,22}$ In the late 1960s and the early 1970s, a few research groups, including the Sandorfy et al., found interesting facts concerning the relative intensities of the bands due to the first overtones of the $\mathrm{OH}$ or $\mathrm{NH}$ stretching modes of free and associated species (e.g., alcohol) in comparison with those of the corresponding fundamentals. ${ }^{21}$ The relative intensity of free $\mathrm{OH}$ or $\mathrm{NH}$ bands is much greater for the overtones than for the fundamentals. Figure 9 compares the MIR spectrum of $0.5 \mathrm{M}$ methanol- $d_{3}$ in $n$-hexane with its corresponding NIR spectrum. ${ }^{40}$ In the MIR spectra, the weak band at $3654 \mathrm{~cm}^{-1}$ is derived from the stretching mode of the free $\mathrm{OH}$ group of methanol- $d_{3}$, and the broad feature in the $3600-3000 \mathrm{~cm}^{-1}$ region arises from the $\mathrm{OH}$ stretching mode of the methanol- $d_{3}$ dimer and oligomers. In contrast to the MIR spectrum, in the NIR spectrum, a band due to the first overtone of the stretching mode of the free $\mathrm{OH}$ group of methanol- $d_{3}$ appears strongly, while bands originating from the first overtone of the $\mathrm{OH}$ stretching mode of the methanol- $d_{3}$ dimer and oligomers are very weak. In general, in the NIR spectrum, a band due to the first overtone of the $\mathrm{OH}(\mathrm{NH})$ 
(a)

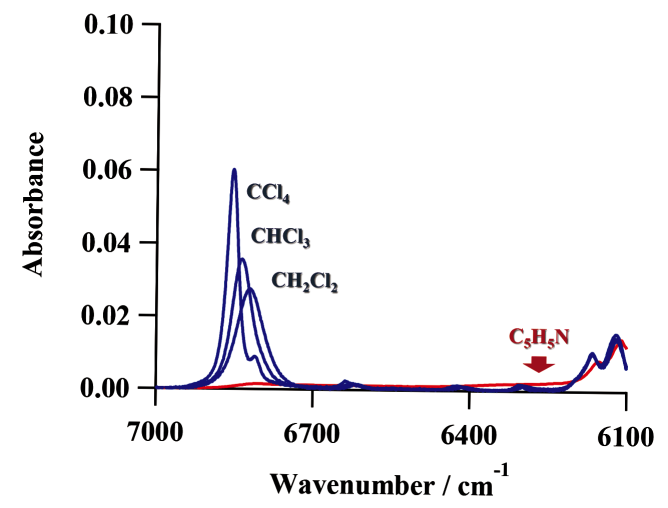

(b)

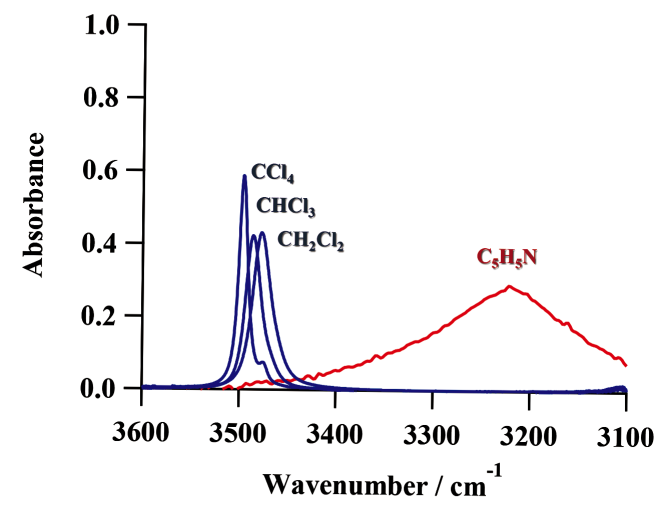

(a')

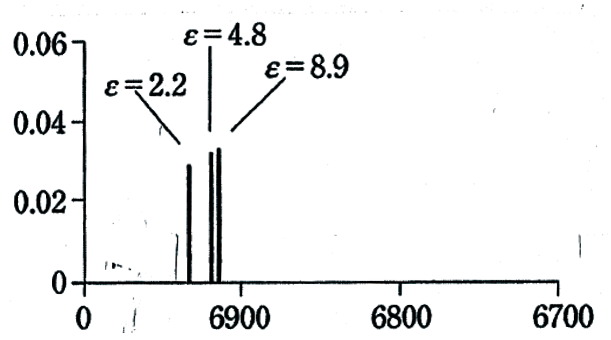

(b')

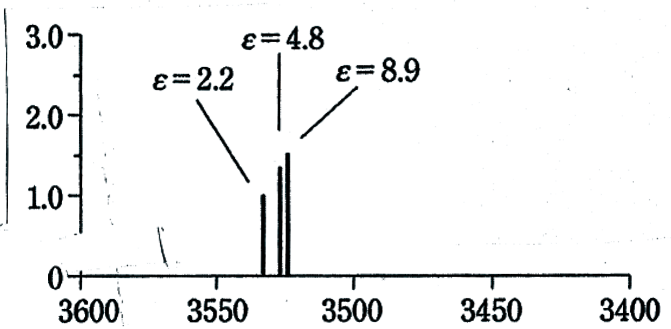

Fig. 10 (a) NIR and (b) MIR spectra of neat pyrrole and pyrrole in $\mathrm{CCl}_{4}, \mathrm{CHCl}_{3}$, and $\mathrm{CH}_{2} \mathrm{Cl}_{2}$. (a') Corresponding calculated NIR and ( $\left.b^{\prime}\right)$ MIR spectra (Reproduced from Ref. 41a with permission).

stretching mode is enhanced owing to the anharmonicity; while, in the MIR spectrum, hydrogen-bonded species exhibit an enhanced $\mathrm{OH}(\mathrm{NH})$ stretching band., ${ }^{70-42}$ It is well known that the integrated intensity of a hydrogen-bonded MIR band is proportional to the square of $\frac{\partial p}{\partial R_{\mathrm{OH}}}$, where $p$ and $R_{\mathrm{OH}}$ are the dipole moment and $\mathrm{OH}$ nuclear distance, respectively. Hence, care must be taken when the band intensity is estimated in both NIR and MIR spectroscopy.

Figure 10 shows the (a) NIR and (b) MIR spectra of neat pyrrole, as well as pyrrole in $\mathrm{CCl}_{4}, \mathrm{CHCl}_{3}$, and $\mathrm{CH}_{2} \mathrm{Cl}_{2}$. ${ }^{41 a}$ The corresponding calculated $\left(a^{\prime}\right)$ NIR and $\left(b^{\prime}\right)$ MIR spectra are also shown in this figure. Notably, the $\mathrm{NH}$ stretching band of pyrrole in $\mathrm{CCl}_{4}, \mathrm{CHCl}_{3}$, and $\mathrm{CH}_{2} \mathrm{Cl}_{2}$ show a lower wavenumber shift in the order of their static relative dielectric constant $(2.2,4.8$, and 8.9, respectively, for $\mathrm{CCl}_{4}, \mathrm{CHCl}_{3}$, and $\mathrm{CH}_{2} \mathrm{Cl}_{2}$ ) with increasing intensity. The increase in intensity is more notable for the fundamental mode than that for the first overtone mode. It has been found that the static relative dielectric constant, $\varepsilon$, of the solvent plays a key role in the solvent dependences of the NIR and MIR spectra. The NH stretching band of pure pyrrole is very broad and enhanced in the MIR spectrum because of the hydrogen bond formation, while the corresponding overtone band is very weak (Fig. 10). ${ }^{41 a}$ Futami et al. ${ }^{41 a}$ successfully reproduced the observed variations due to changes in the dielectric constant, $\varepsilon$, in their calculated data in terms of wavenumber and intensity (Figs. 10( $\left.a^{\prime}\right)$ and $10\left(b^{\prime}\right)$ ).

Futami et al. ${ }^{41 \mathrm{a}, \mathrm{b}}$ also compared the effects of hydrogen bonds and those of solvents on wavenumbers and intensities of the fundamental and first overtone of the $\mathrm{NH}$ stretching mode of pyrrole via NIR and MIR spectroscopy. It was found that only NIR spectroscopy can distinguish between the spectral changes that are induced by hydrogen bond formation and those by solvent changes. Gonjo et al., Hofer et al., and Morisawa et al. investigated the hydrogen bonding of alcohols and phenol derivatives using higher-order overtones and quantum chemical calculations. ${ }^{1 \mathrm{c}, 39,42 \mathrm{a}, \mathrm{b}}$

3.2.2 Quantum chemical calculations in NIR spectroscopy

In contrast to the MIR region, the harmonic oscillator approach cannot provide any information about the transitions relevant to the NIR region. ${ }^{43,44}$ This has been the basic limiting factor. Thus, anharmonic calculation is indispensable for the theoretical investigation of NIR spectroscopy; however, it requires considerable computational time. ${ }^{43,44}$ Hence, investigations of the entire NIR spectra, based on quantum chemical calculations, have been very rare until recently because of the markedly increased complexity in the theoretical descriptions of the anharmonic effects. ${ }^{43}$ One cannot describe overtones by harmonic approximation. In addition, the quantum harmonic calculation, which does not include mode coupling, cannot treat the combination modes. Therefore, any theoretical approach that is applicable to NIR spectroscopy must be beyond harmonic approximation. Therefore, one can find only a few comprehensive anharmonic approaches. ${ }^{43,45,46}$ The vibrational self-consistent field (VSCF) approach, a variational method, is one of the first widely implemented anharmonic methods. VSCF is useful in most cases; however, in its original form, it is not very efficient. Consequently, techniques for improving this method have been proposed. ${ }^{43}$

Recently, using anharmonic calculations, Bec et al. ${ }^{47-49}$ succeeded in performing quantum chemical calculations of NIR spectra of not only simple organic compounds, such alcohols and fatty acids, but also of more complicated molecules, such as long chain fatty acids (LCFAs). Figure 11 depicts the experimental and calculated NIR spectra of low concentration $(0.005 \mathrm{M})$ methanol in $\mathrm{CCl}_{4}$ in the $7500-3700 \mathrm{~cm}^{-1}$ region. ${ }^{47}$ 


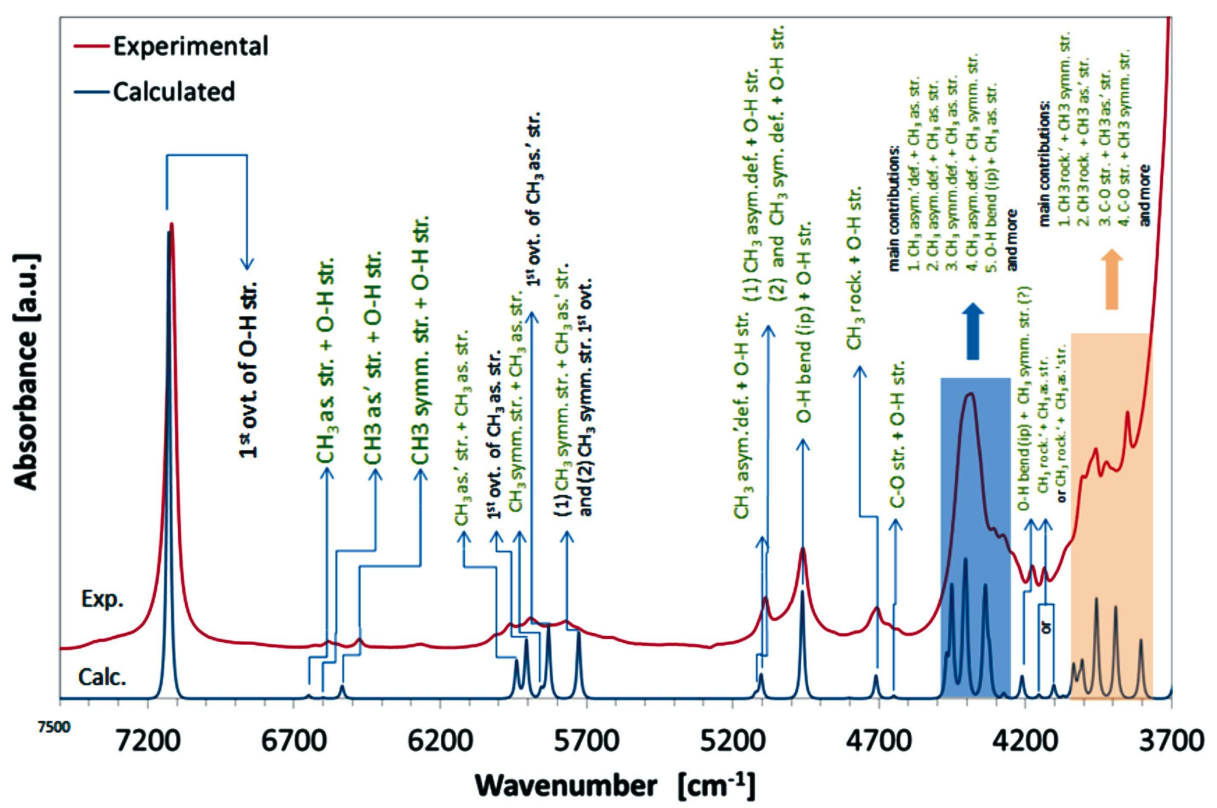

Fig. 11 Comparison of an experimental NIR spectrum of low concentration $(0.005 \mathrm{M})$ methanol in $\mathrm{CCl}_{4}$ and a calculated NIR spectrum; GVPT2 calculations on CPCM-B2PLYP-D/SNST level of theory were used here (Reproduced from Ref. 47 with the permission).

The agreement between the experimental and calculated spectra is very good. One can easily assign the band at $7130 \mathrm{~cm}^{-1}$ to the first overtone of the $\mathrm{OH}$ stretching mode of free methanol. For the other bands, the quantum chemical calculations are used for band assignments; note that many of the bands are assigned to the combination modes. ${ }^{47}$

In this review, I have reported some details on the NIR spectra simulations using anharmonic calculations of the NIR spectra of saturated and unsaturated LCFAs. ${ }^{48}$ Grabska et al. ${ }^{48}$ used fully anharmonic spectral simulations for arachidic acid, palmitic acid, stearic acid, linoleic acid, linolenic acid, and oleic acid. The spectral features of these LCFAs were accurately reproduced by the deperturbed vibrational second-order perturbation theory (DVPT2) throughout a wide NIR region $\left(8000-4000 \mathrm{~cm}^{-1}\right)$, and detailed band assignments were then provided. ${ }^{48}$ The effect of the saturation of the alkyl chain and the dependency of the number of $\mathrm{C}=\mathrm{C}$ bonds were clearly reflected in the simulated NIR spectra.

Figure 12 compares the experimental $\left(0.05 \mathrm{M}\right.$ in $\left.\mathrm{CCl}_{4}\right)$ and simulated NIR spectra for (a) arachidic acid, (b) palmitic acid, (c) stearic acid, (d) linoleic acid, (e) $\alpha$-linolenic acid, and (f) oleic acid in the $7500-4000 \mathrm{~cm}^{-1}$ region. ${ }^{48}$ The agreement between the calculated and experimental NIR spectra of LCFAs is satisfactory. Notably, the saturated and unsaturated LCFAs can be distinguished clearly by the two spectral sub-regions $\left(6000-5500\right.$ and $\left.4700-4500 \mathrm{~cm}^{-1}\right)$, which are specific to the type of alkyl chain. The spectra of all the investigated fatty acids show a well-resolved band at approximately $7000 \mathrm{~cm}^{-1}$. This is due to the first overtone of the stretching mode $(2 v \mathrm{OH})$ of the non-bonded $\mathrm{OH}$ group of monomeric LCFAs. The broadening of the $2 v \mathrm{OH}$ band occurs because the LCFAs contain a large number of conformers. The calculated $2 v \mathrm{OH}$ wavenumber changes among the studied molecules, unlike the experimental peak position. This will be discussed later.

The $6000-5500 \mathrm{~cm}^{-1}$ region contains several bands derived from the first overtones and combinations of $\mathrm{CH}_{3}, \mathrm{CH}_{2}$, and $\mathrm{CH}$ stretching modes. The spectra of saturated fatty acids are more similar to each other than those of the unsaturated fatty acids (Fig. 12), revealing consistency in the structure-spectra relationship and the influence of the $\mathrm{C}=\mathrm{C}$ bond. In this region, two broad features are observed. For the saturated LCFAs, the peak maxima are located at the same positions, i.e., 5785 and $5676 \mathrm{~cm}^{-1}$. The position of the lower wavenumber band of the unsaturated fatty acids are almost the same, while that of the higher wavenumber band varies significantly; for oleic acid, it appears at $5793 \mathrm{~cm}^{-1}$; for linoleic acid at $5836 \mathrm{~cm}^{-1}$; and for linolenic acid at $5841 \mathrm{~cm}^{-1}$. In all the cases, these peaks show a higher wavenumber shift compared to the corresponding peaks of the saturated LCFAs. Grabska et al. ${ }^{48}$ further explored the mode contributions to the NIR spectra of saturated and unsaturated LCFAs. In the $6200-5500 \mathrm{~cm}^{-1}$ region, the contribution of the overtones is much smaller than that of combination modes for both saturated and unsaturated LCFAs. In particular, in the spectra of the saturated fatty acids, the relative contributions from the overtones are always lower. In the spectra of unsaturated fatty acids, the first overtone bands have stronger intensities in this region. According to our calculation for saturated compounds, the contribution from the overtones is below only $6 \%$, while in the case of unsaturated ones, it is between 5 and $17 \%$. Note that the simulated and experimental NIR spectra show some discrepancy in the $6200-$ $5400 \mathrm{~cm}^{-1}$ region (Fig. 12). The possible reason for this discrepancy may be the high anharmonicity of the $\mathrm{C}-\mathrm{H}$ stretching vibrations, whose first overtones and binary combinations dominate the region.

This anharmonic calculation revealed that DVPT2 method is useful for the calculation of complicated molecules, such as LCFAs; however, this method does not yield satisfactory results for modes with high anharmonicity, for example, the deviation of the band due to the first overtone of $\mathrm{OH}$ stretching mode of a monomer.

3.2.3 NIR imaging using novel instruments

NIR imaging is an in situ and non-destructive analysis method; it allows the extraction of information about the spatial 

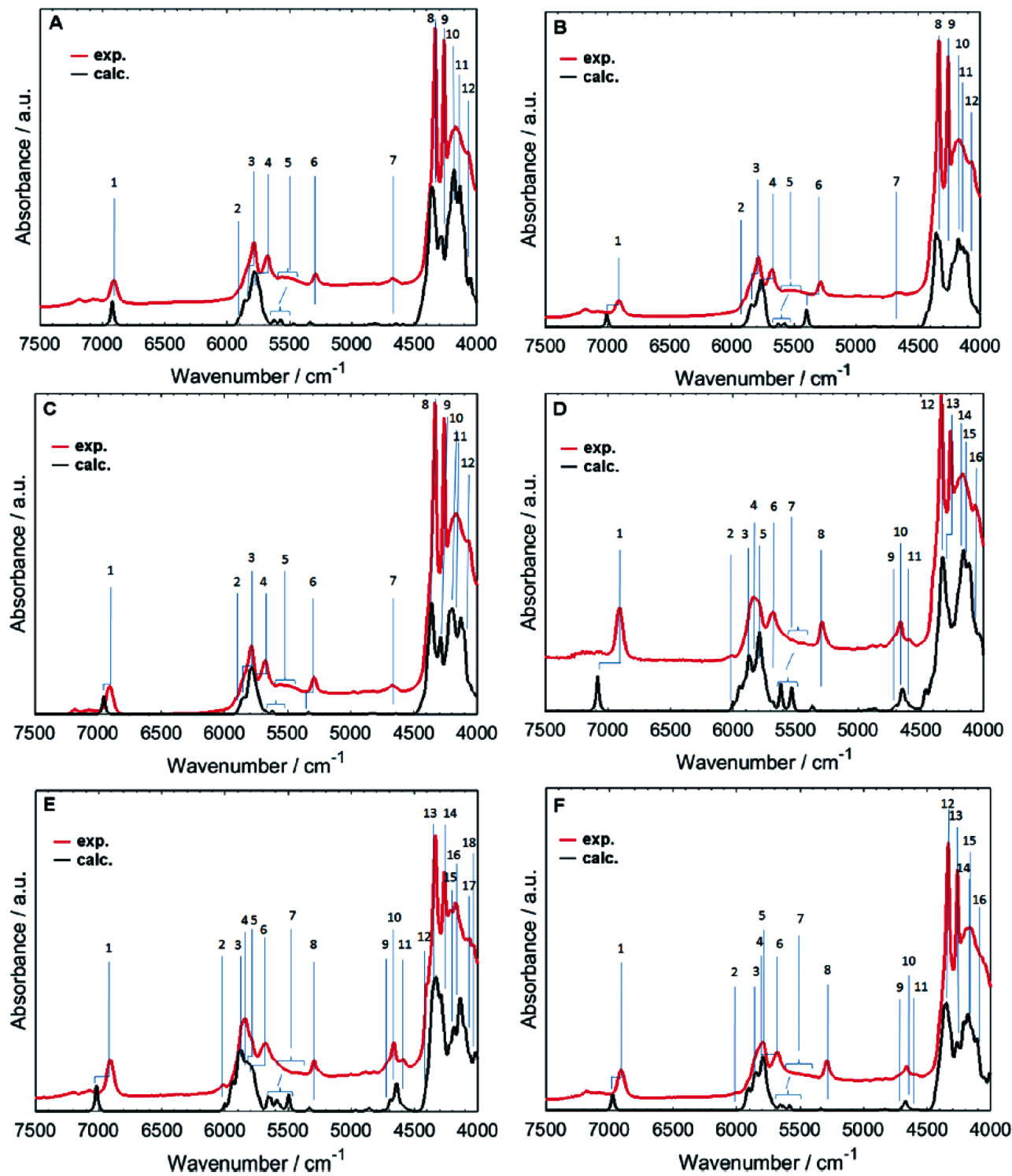

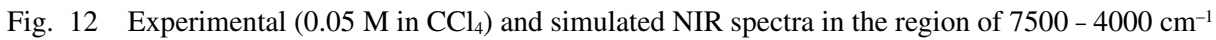
of (a) arachidic acid, (b) palmitic acid, (c) stearic acid, (d) linoleic acid, (e) $\alpha$-linolenic acid, and (f) oleic acid (Reproduced from Ref. 48 with permission).

distribution of chemical components, even from thick materials. ${ }^{6,750-55}$ It also enables non-contact and optical fibreaided analysis. Similar to the case of conventional NIR spectroscopy, NIR spectral images can be obtained using different modalities, such as transmission, reflectance, transflectance, or interactance. Using NIR imaging, one can probe an aqueous solution ${ }^{7,50}$ or organic solvent ${ }^{51,52}$ dispersion more easily, compared to using MIR imaging. NIR imaging has been utilised for various applications. Further, this technique has been used to image various types of materials, such as polymers, biomedical samples, foods, and pharmaceutical tablets. ${ }^{6,7,50-55}$

In recent years, three types of innovative NIR imaging systems have been developed: ${ }^{50,51,53-55}$ (i) a portable NIR imaging $(151 \times$ $93 \times 120 \mathrm{~mm}^{3}$ ) system with an InGaAs-photodiode array (D-NIRs; Yokogawa Electric Co.);50,51a (ii) a high-speed, widearea $\left(150 \times 200 \mathrm{~mm}^{2}\right)$, and micro-area monitoring NIR instrument with a highly sensitive NIR camera (Compovision, Sumitomo Electric Industries Ltd.), ${ }^{51 b, 55}$ and (iii) an imaging- type 2D Fourier spectroscopy (ITFS) system (proposed by Ishimaru and commercialised by Aoi Electronics Co. Ltd.). ${ }^{53}$

Ishigaki et al. ${ }^{54}$ explored the growth process of fertilised eggs from Japanese medaka fish (Oryzias laptipes) non-invasively using Compovision. Using Compovison, NIR imaging of a medaka egg can be obtained within a few seconds. Hence, the egg remains alive during the imaging. Figure 13 shows the second derivatives of the NIR spectra of four types of medaka eggs in the $5500-4100 \mathrm{~cm}^{-1}$ region: one group of eggs activated by fertilisation (normal), and three groups of eggs not activated because embryogenesis stopped or not started, culturing under cold temperature (dead_1), instant freezing (dead_2), or lack of fertilisation (UF). The broad features in the $5300-5100 \mathrm{~cm}^{-1}$ region are due to the water included in the eggs; the more intense peak at approxiamtely $5250 \mathrm{~cm}^{-1}$ is assigned to weak hydrogen-bonded water (WHB) species, while the weaker one in the $5200-5100 \mathrm{~cm}^{-1}$ region originates from strong hydrogenbonded water (SWB) species. Several weak features in the $5000-4100 \mathrm{~cm}^{-1}$ region arise from proteins and lipids in the 
eggs. A benefit of NIR spectroscopy is that it is possible to monitor the structural and concentration changes in water and proteins simultaneously. This feature is not available in MIR and Raman spectroscopy. Figure 14 displays (top) visible images of the four types of medaka eggs and (bottom) NIR images constructed by plotting the ratio of the second derivative intensities of the water bands, which are defined as $I_{5170} / I_{5250} .{ }^{54}$ To visualise the bioactivity of the fish eggs, Ishigaki et al. ${ }^{54}$ investigated the distributions of SHB and WHB species of water in the eggs. The reddish colour in Fig. 14 indicates that the ratio of SHB was higher. Therefore, in the non-activated eggs, the proportion of SHB water $\left(\sim 5170 \mathrm{~cm}^{-1}\right)$ was higher than that in the activated eggs. Moreover, the distributions of SHB and WHB are significantly different among the non-activated eggs.

Ishigaki et al..$^{53}$ also performed non-staining blood flow imaging of the medaka eggs using an optical interface due to Doppler shift (ITFS).

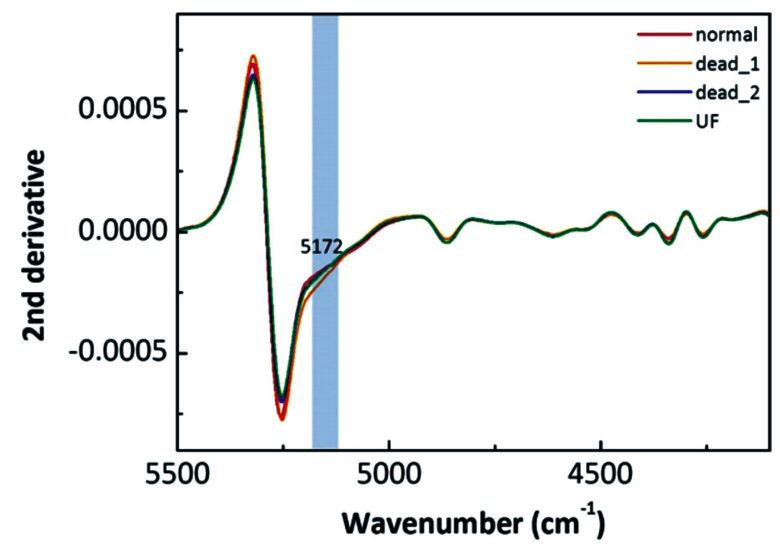

Fig. 13 The second derivatives of NIR spectra in the 5500$4100 \mathrm{~cm}^{-1}$ region of four types of medaka eggs; one group of eggs activated by fertilization (normal), and three groups of eggs not activated because embryogenesis was stopped or not started by culturing under cold temperature (dead_1), instant freezing (dead_2), or lack of fertilization (UF).
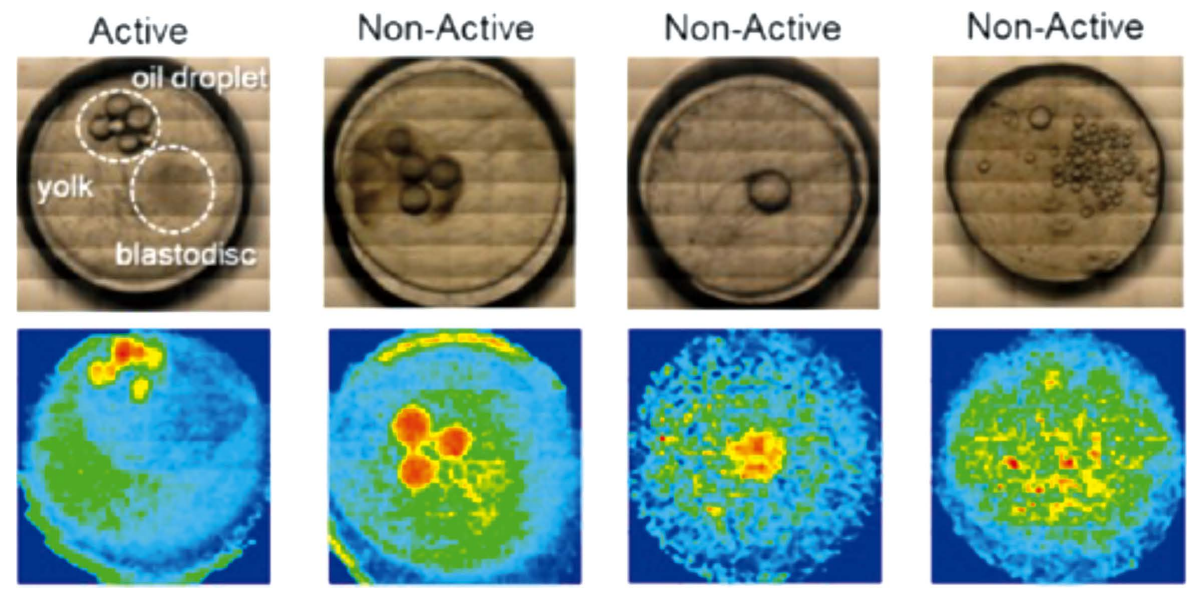

Fig. 14 (top) Visible images of the four types of medaka eggs and (bottom) NIR images constructed by plotting the ratio of the second derivative intensities of the water bands defined as $I_{5170} / I_{5250}$ (Reproduced from Ref. 54 with permission). 


\subsection{Characteristics and advantages of FIR or THz spectroscopy}

There are significant differences in the force constants and reduced masses between the FIR or THz and MIR regions. We can assume the following equation for an intermolecular vibration of molecular chains and a vibration of functional group consisting of two atoms for FIR or $\mathrm{THz}$ and MIR spectroscopy, respectively:

$$
f=1 / 2 \pi \sqrt{k} / \mu
$$

where $f, k$, and $\mu$ represent the vibrational frequency, force constant, and reduced mass, respectively. The force constant is 100 to 1000 times larger for MIR spectroscopy, while the reduced mass is 100 to 1000 times larger for FIR or $\mathrm{THz}$ spectroscopy. Consequently, the frequencies of molecular vibrations are approximately 100 times larger in the MIR spectroscopy. Thus, MIR spectroscopy detects the molecular vibrations of functional groups, while FIR or $\mathrm{THz}$ spectroscopy detects the intermolecular vibrations of the molecular assemblies.

\subsection{Applications of FIR or THz spectroscopy}

In this region, vibrational spectroscopy has been recently used to investigate the structures, intermolecular interactions, hydrogen bondings, hydrations, and dynamics of various types of molecules, materials, and tissues. ${ }^{10-12,56-61}$ In this review, I have focused on the applications of hydrogen bonding, intermolecular interactions, and higher-order structures of polymers. ${ }^{56-61}$ Moreover, the application of $\mathrm{THz}$ imaging to the study of distributions of crystallinity and crystalline orientation in a poly ( $\varepsilon$-caprolactone) film is introduced. ${ }^{61}$

4.2-1 FIR or THz spectroscopy, low-frequency Raman spectroscopy, and quantum chemistry studies of polymers with strong and weak hydrogen bonds

Low-frequency vibrational modes of polymers can provide unique information about the non-covalent bonds among the polymer chains, which are often crucial for understanding the properties of polymers. However, the developments in FIR and low-frequency Raman spectroscopy investigations of polymers are rather slow. There are two major reasons for this. In addition to the insufficient advances in instrumentation, the lack of a proper band assignment method has been a serious problem. Therefore, our group has set up a new strategy for low-frequency vibrational spectroscopic studies of polymers. ${ }^{56-61}$ It uses FIR, $\mathrm{THz}$, and low-frequency Raman spectroscopy, including polarised measurements, and performs quantum mechanical calculations combined with the Cartesian coordinate tensor transfer method for band assignment. In the calculations, we considered how to calculate the spectra of molecules involving intermolecular interactions and those with high molecular weights.

Yamamoto et al. conducted FIR or $\mathrm{THz}$ and Raman spectroscopy and quantum chemical calculations of nylon $6^{60}$ and various biodegradable polymers, such as $\mathrm{PHB}^{56}$ and poly(glycolic acid) (PGA). ${ }^{57}$ Nylon 6 has strong hydrogen bonds, whereas PHB and PGA have weak hydrogen bonds. Our research aims are to explore the intermolecular interactions, higher-order structure, and thermal behaviours of these polymers. Moreover, I have discussed the similarities and differences in the low-frequency vibrational spectra among the above polymers. ${ }^{60}$ The intermolecular interactions among the polymer chains are considered explicitly in our quantum mechanical calculations. We attempted to determine the type of new information that low-frequency vibrational spectroscopy can provide about a polymer, which MIR and Raman spectroscopy cannot yield.

\subsubsection{FIR, THz, low-frequency Raman, and quantum} chemical study of nylon 6

Yamamoto et al. ${ }^{60}$ investigated the structure and thermal behaviour of nylon 6 using FIR, low-frequency Raman spectroscopy, and quantum chemical calculations. Notably, nylon 6 shows crystalline polymorphism, and its $\alpha$ form is the most stable. ${ }^{60}$ The monoclinic crystal of the $\alpha$ form includes hydrogen bonds along the $a$-axis, forming a sheet structure among the neighbouring antiparallel chains. After the glass transition at $\sim 54^{\circ} \mathrm{C}$, the lattice lengths of the $a$ - and $c$-axes gradually became closer to each other with an increase in temperature, and finally became nearly equal after the Brill transition at $\sim 160^{\circ} \mathrm{C} .^{60}$

Figures 15(a) and 15(b) show temperature-dependent FIR spectra and their second derivatives in the $350-50 \mathrm{~cm}^{-1}$ region of nylon 6 measured over a temperature range of $29-194^{\circ} \mathrm{C} .60$ The FIR spectrum at $29^{\circ} \mathrm{C}$ exhibits characteristic crystalline bands at 294, $\sim 222$, and $\sim 111 \mathrm{~cm}^{-1}$ that almost disappear at $202^{\circ} \mathrm{C}$, which is close to the melting point. In the $125-80 \mathrm{~cm}^{-1}$ region, one can observe a broad feature in the original spectrum; however, the second derivative spectrum clearly identifies two bands at 112 and $98 \mathrm{~cm}^{-1}$. With increasing temperature, the intensity at $112 \mathrm{~cm}^{-1}$ decreases. Figure 16(a), (b), and (c), respectively, plot the FIR intensity of nylon 6 at 294, 222, and $111 \mathrm{~cm}^{-1}$ versus the temperature. ${ }^{61}$ The intensity of the $111 \mathrm{~cm}^{-1}$ peak changes negligibly until $\sim 60^{\circ} \mathrm{C}$, which corresponds to the temperature of glass transition of nylon 6 at $\sim 54^{\circ} \mathrm{C}$. Above $\sim 60^{\circ} \mathrm{C}$, the intensity decreases linearly, and becomes constant above $\sim 170^{\circ} \mathrm{C}$. This temperature is close to the Brill transition of nylon 6 at $\sim 160^{\circ} \mathrm{C}$. Notably, after the glass transition, the lattice lengths of the $a$ - and $c$-axes of an $\alpha$-form nylon 6 gradually become closer to each other and finally reach unity after the Brill transition. ${ }^{60}$ The FIR intensity at $111 \mathrm{~cm}^{-1}$ demonstrates a similar tendency. Hence, the intensity at $111 \mathrm{~cm}^{-1}$ may be used as an indicator of the lattice length of nylon 6 in the $\alpha$ form. The intensities of bands in a region of $222-192 \mathrm{~cm}^{-1}$ decrease as the temperature increases. ${ }^{60}$ The temperature dependence of the intensity at $222 \mathrm{~cm}^{-1}$ (Fig. 16) shows a transition temperatures of $\sim 50^{\circ} \mathrm{C}$ (glass) and $\sim 160^{\circ} \mathrm{C}$ (Brill). The strong band at $294 \mathrm{~cm}^{-1}$ becomes broader and weaker as the temperature increases; however, the peak height decreases only gradually, and does not yield any clear transition (Fig. 16).

Temperature-dependent Raman spectra of nylon 6 in $\alpha$-form, measured over a temperature range of $22-210^{\circ} \mathrm{C}$, are shown in Fig. 17(a). ${ }^{60}$ The second derivative spectra are shown in Fig. 17(b). The second derivative spectra clearly demonstrate that there are two bands at 106 and $100 \mathrm{~cm}^{-1}$, and that the relative intensity of these two bands changes with increasing temperature. When the temperature increased to $210^{\circ} \mathrm{C}$, which is close to the melting point of the sample $\left(217^{\circ} \mathrm{C}\right.$ measured by DSC), the observed peaks become much weaker and broader (Fig. 17(a)), indicating that the two peaks are crystalline bands. They measured polarised Raman and FIR spectra of nylon 6 in $\alpha$ form at at $25^{\circ} \mathrm{C}^{60}$ Both strong Raman band at $102 \mathrm{~cm}^{-1}$ and FIR band at $\sim 110 \mathrm{~cm}^{-1}$ are polarised perpendicularly to the chain axis, suggesting the correspondence of these Raman and FIR bands.

Figure 18(a) and (b) compares the experimental (top) and calculated (bottom) Raman and FIR spectra, respectively, of nylon 6 in the $\alpha$-form. ${ }^{60}$ The calculations are performed using a fragment methodology at the CAM-B3LYP-GD3BJ/6-311 $++\mathrm{G}^{* *}$ level of theory. Based on the peak positions and polarisation directions, the experimental Raman bands at 365 , 
(a)

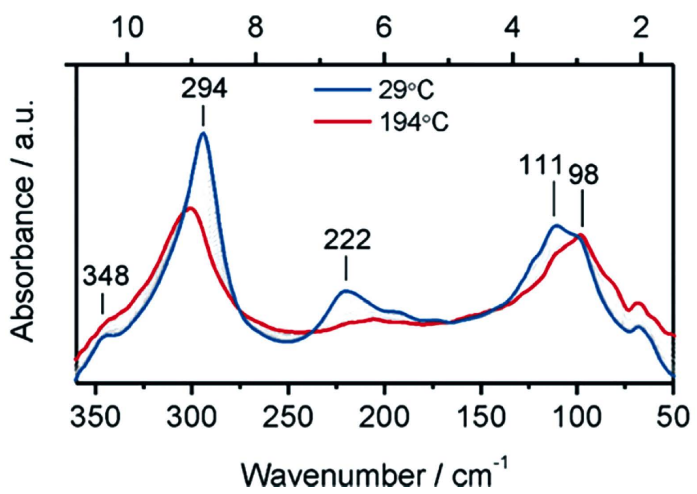

(b)

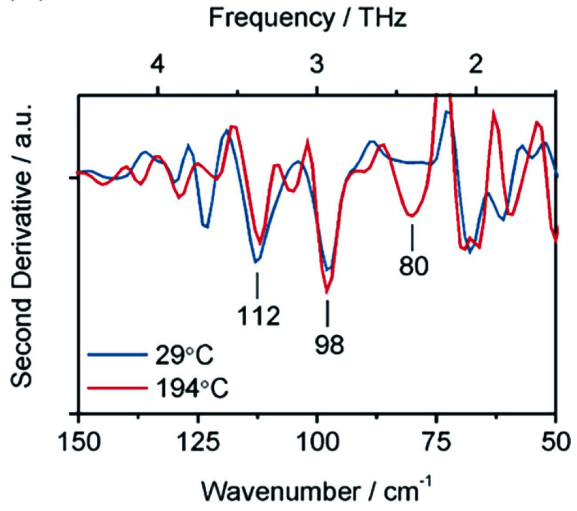

Fig. 15 (a) Temperature-dependent FIR spectra and (b) their second derivatives in the $350-50 \mathrm{~cm}^{-1}$ region of nylon 6 obtained in a temperature range of 29 to $194^{\circ} \mathrm{C}$ (Reproduced from Ref. 61 with permission).

(a)

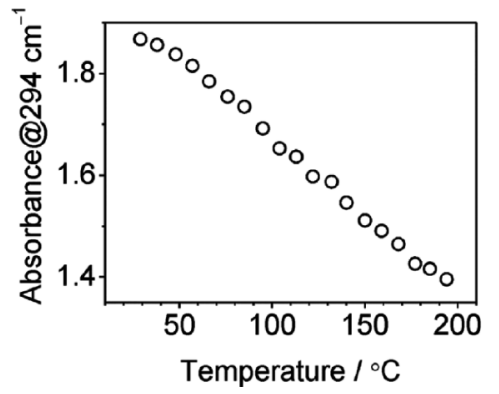

(b)

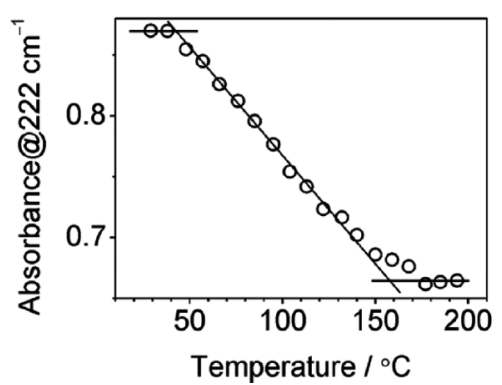

(c)

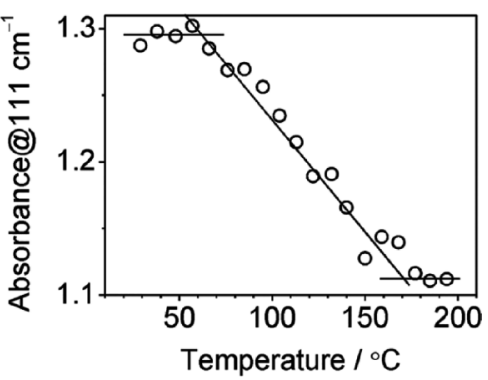

Fig. 16 Plot of FIR intensity of nylon 6 at (a) 294, (b) 222, and (c) $111 \mathrm{~cm}^{-1}$ versus temperature (Reproduced from Ref. 60 with permission).

(a)

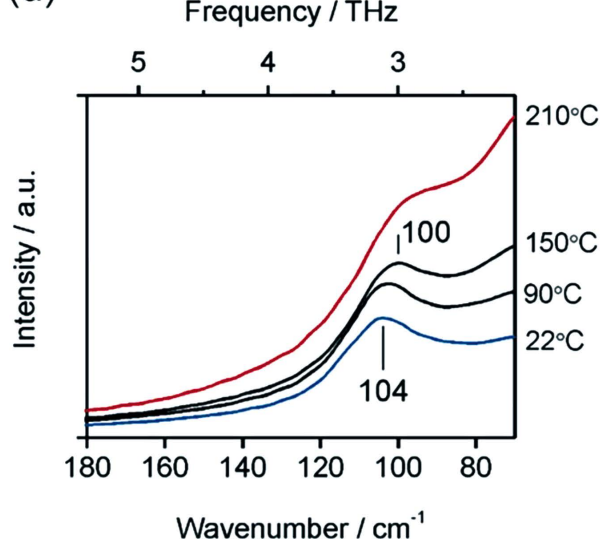

(b)

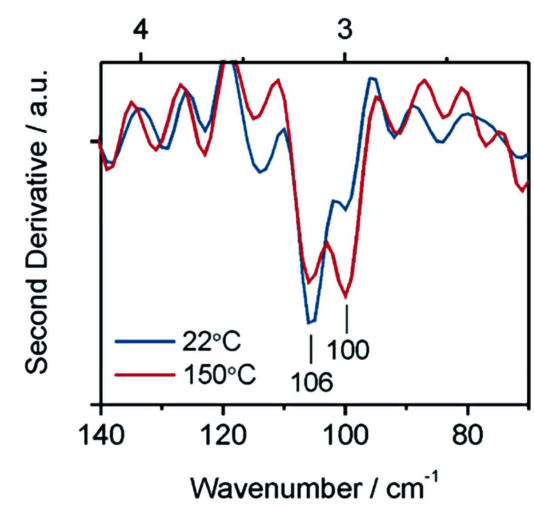

Fig. 17 (a) Temperature-dependent Raman spectra and (b) their second derivative spectra of nylon 6 in $\alpha$-form measured in a temperature range of $22-210^{\circ} \mathrm{C}$ (Reproduced from Ref. 60 with permission).

289 , and $213 \mathrm{~cm}^{-1}$ may be attributed to the calculated bands at 365,273 , and $\sim 210 \mathrm{~cm}^{-1}$, respectively. In the lower wavenumber region, perpendicularly polarised strong Raman bands are calculated at 127, 108, and $89 \mathrm{~cm}^{-1}$, which correspond to the intense experimental band at $\sim 104 \mathrm{~cm}^{-1}$. It is very likely that the calculated strongest band at $108 \mathrm{~cm}^{-1}$ corresponds to the experimental band at $104 \mathrm{~cm}^{-1}$.

The experimental and calculated FIR spectra are in good agreement with each other in terms of both spectral shapes and polarisation directions. The experimental FIR bands at 294 and $222 \mathrm{~cm}^{-1}$, which are calculated at 274 and $216 \mathrm{~cm}^{-1}$, respectively, are consistent with the polarisation directions. The lower 
(a)

Frequency / THz

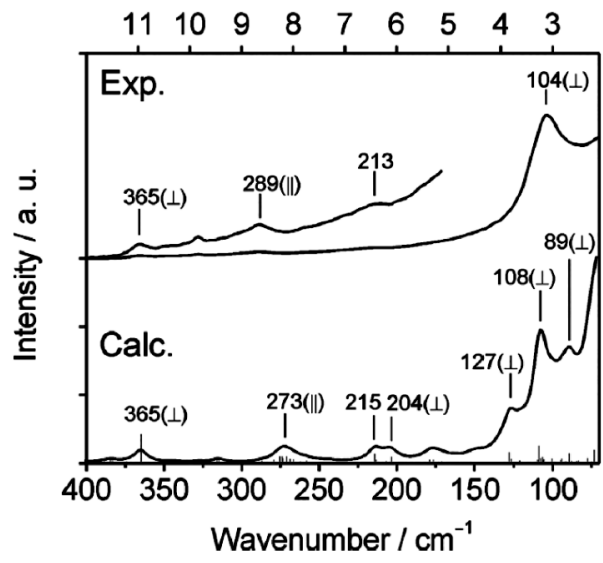

(b)

Frequency / THz

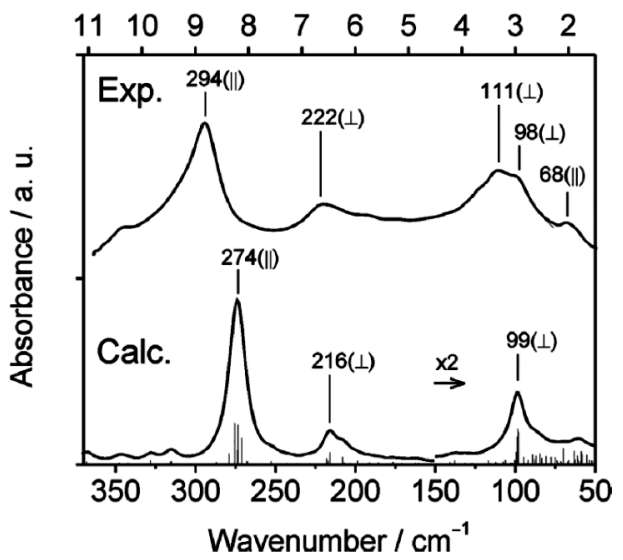

Fig. 18 (a, b) The experimental (top) and calculated (bottom) (a) Raman and (b) FIR spectra of nylon 6 in $\alpha$-form (Reproduced from Ref. 60 with permission).

(a)

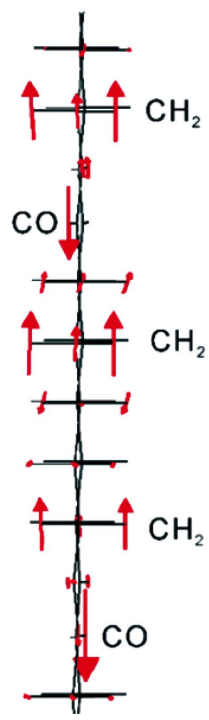

(b)

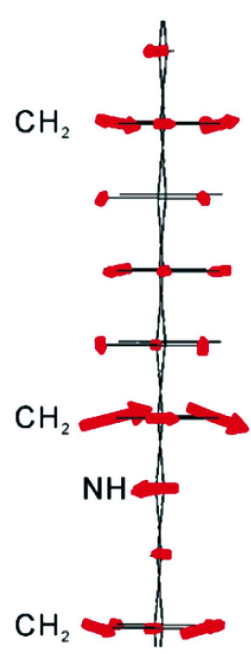

(c)

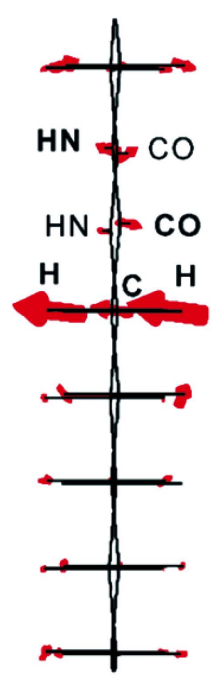

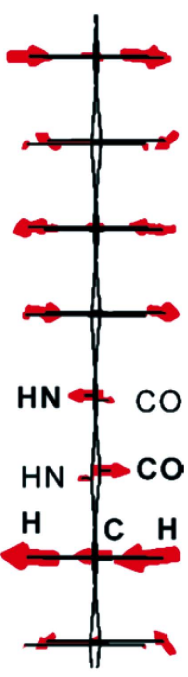

Fig. 19 Atomic motions of the FIR active modes of the nylon 6 in $\alpha$ form calculated for the (a) 247, (b) 216, and (c) $99 \mathrm{~cm}^{-1}$ bands viewed from the origin of $a$ axis (Reproduced from Ref. 60 with permission).

wavenumber region is rather complicated in the experimental spectrum; however, in the calculation, a distinct FIR band is observed at $99 \mathrm{~cm}^{-1}$ with perpendicular polarisation and a weak shoulder feature at $\sim 80 \mathrm{~cm}^{-1}$. Based on the polarisation and intensities, these calculated bands correspond to the experimental bands at $\sim 111$ and $\sim 98 \mathrm{~cm}^{-1}$.

Figure 19(a), (b), and (c), respectively, illustrates the atomic motions of the FIR active modes of nylon 6 in the $\alpha$ form calculated for the 247, 216, and $99 \mathrm{~cm}^{-1}$ bands viewed from the origin of $a$-axis. ${ }^{60}$ Figure 19(c) shows that the calculated atomic motions of the FIR band at $99 \mathrm{~cm}^{-1}$ consist of a torsional motion of the methylene groups and the transverse mode of amide groups, in which the $\mathrm{NH}$ and $\mathrm{O}$ atoms oscillate out of the amide plane. As discussed later, the amide groups dominate the intensity of the $99 \mathrm{~cm}^{-1}$ band. The translational motion of the amides may become restricted in the polymer chains due to the large mass ${ }^{60}$ The torsional nature of this band can explain why its intensity is sensitive to changes in the lattice length (Fig. 16). The calculated Raman band at $108 \mathrm{~cm}^{-1}$ depicts atomic motions similar to the FIR band at $99 \mathrm{~cm}^{-1}$, torsion of methylene groups, and transverse motion of the amide groups. The experimental FIR band at $\sim 220 \mathrm{~cm}^{-1}$ corresponds to the calculated band at $216 \mathrm{~cm}^{-1}$, the assignment of which is a torsional vibration of methylene groups and out-of-plane motion of $\mathrm{NH}$ groups (Fig. 19). ${ }^{60}$ This band intensity gives rise to the two transition points as the temperature increases. Thus, naturally, this torsional mode is sensitive to the lattice length of nylon 6 .

To estimate the contribution of the amide groups to the spectral intensities, Yamamoto et al. ${ }^{60}$ calculated the spectra by zeroing the atomic tensors of the amide groups and compared them to the exact calculations (Fig. 20). Except for the Raman band at $270 \mathrm{~cm}^{-1}$, the intensities of all the other main Raman 
(a)

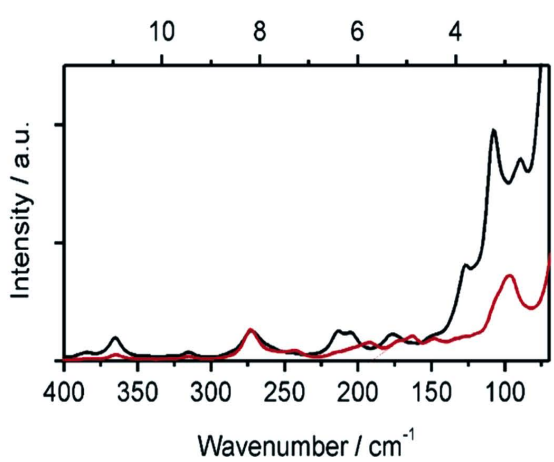

(b)

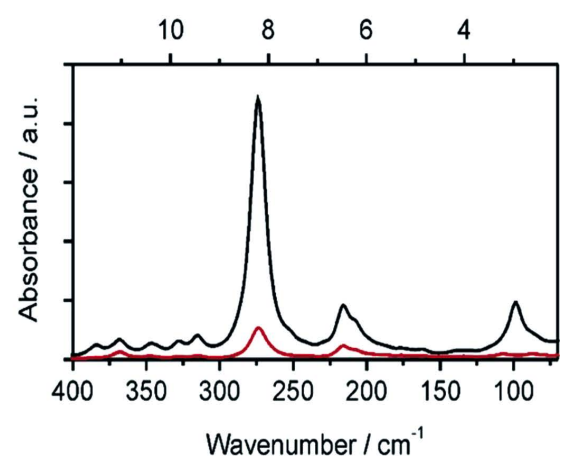

Fig. 20 The spectra with zeroing out atomic tensors of the amide groups and compared them to the exact calculations (Reproduced from Ref. 60 with permission).

and FIR bands significantly reduced via zeroing, indicating the dominant contribution of the amide groups to the low-frequency band intensities. Note that the FIR band at $\sim 100 \mathrm{~cm}^{-1}$ disappears almost perfectly, suggesting that the $\sim 100 \mathrm{~cm}^{-1}$ band is highly sensitive to the chemical environment of the amides. Interestingly, the atomic motions of the FIR band at $99 \mathrm{~cm}^{-1}$ are dominated by the methylene group; however, its absorption intensity is governed by the amide groups.

Yamamoto et al. ${ }^{56,57,60}$ compared the FIR or THz, lowfrequency Raman spectra, and quantum chemical calculations of crystalline polyesters, PHB and PGA, with those of nylon 6, which is a polyamide. They found a significant resemblance in the low-frequency vibrational bands in terms of both frequencies and intensities. Nylon 6, PHB, and PGA showed a specific peak around $100 \mathrm{~cm}^{-1}$, which is assigned to torsional motions containing ester or amide groups with perpendicular polarisation. These low-frequency bands may be used to probe the interchain interactions and/or lattice length of the polymers, which could be an advantage of low-frequency vibrational spectroscopy. The combined method, including FIR or THz spectroscopy, Raman spectroscopy, and quantum mechanical calculations, has become a powerful tool for assigning bands in the low-frequency vibrational spectra of polymers, which aids in exploring their origins.

4.2.3 Studying the distribution of crystallinity and crystalline orientation in a poly( $\varepsilon$-caprolactone) film via THz imaging

The distribution of crystallinity and crystalline orientation of a poly( $\varepsilon$-caprolactone) (PCL) film was investigated using $\mathrm{THz}$ imaging. ${ }^{61} \mathrm{THz}$ images were developed using the intensity ratio of the two peaks at 1.42 and $2.03 \mathrm{THz}$, owing to the crystalline modes that were parallel and perpendicular to the $c$-axis $\left(I_{\perp} / I_{/ l}\right)$ of the PCL film. It was found from the $\mathrm{THz}$ images that the distribution of crystallinity and crystalline orientation change significantly in the different regions in the PCL film, although the corresponding optical image did not show this inhomogeneity. This study revealed that $\mathrm{THz}$ imaging is beneficial for characterising the physical properties of semi-crystalline polymers. One of the advantages of $\mathrm{THz}$ imaging is that a wider area (a few $\mathrm{cm} \times \mathrm{a}$ few $\mathrm{cm}$ ) can be investigated by this method, as compared to ordinary MIR imaging.

Figure 21 shows the $\mathrm{THz}$ absorption spectra of a PCL film measured in the temperature range $30-70^{\circ} \mathrm{C} .{ }^{61}$ Note that the two major peaks at 1.42 and $2.03 \mathrm{THz}\left(47\right.$ and $\left.67 \mathrm{~cm}^{-1}\right)$ shift with temperature. Sato et al. ${ }^{61}$ ascribed the shifts to the large anharmonicity of the vibrational potential. The intensity of both<smiles>CCCCCCC(=O)OCC</smiles>

(a)
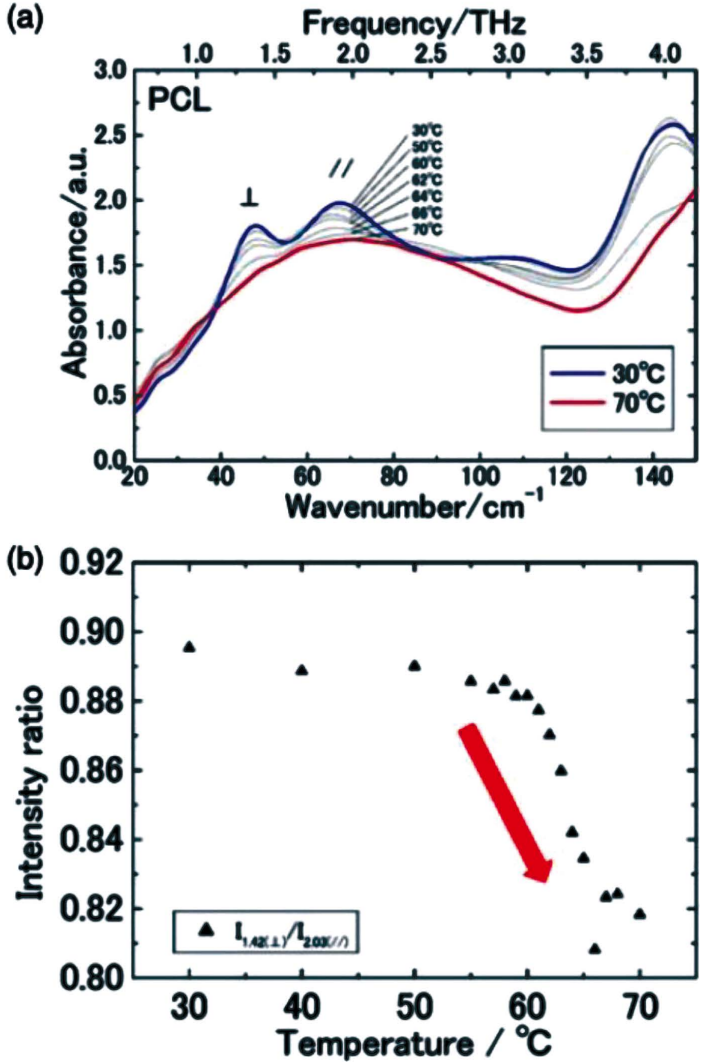

Fig. 21 The THz absorption spectra of a PCL film collected in the temperature range $30-70^{\circ} \mathrm{C}$ (Reproduced from Ref. 61 with permission).

peaks decrease with increasing temperature; thus, they are assigned to the crystalline modes. The temperature-dependent variations in the intensity ratio of the two peaks $\left(I_{1.42} / I_{2.03}\right)$ are plotted in Fig. 21(b). ${ }^{61}$ The intensity ratio shows a large change in the vicinity of the PCL melting temperature $\left(60^{\circ} \mathrm{C}\right)$ (Fig. 21(b)), implying that the intensity ratio $\left(I_{1.42} / I_{2.03}\right)$ is 


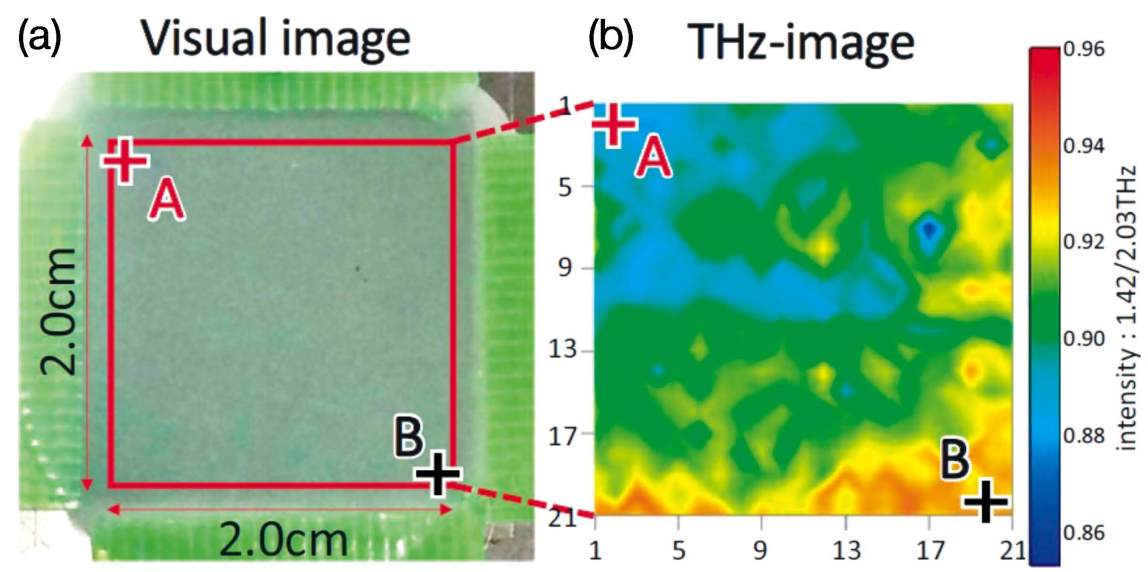

Fig. 22 (a) The visual image of a PCL film and (b) the corresponding THz transmission image developed using $I_{1.42} / I_{2.03}$ (Reproduced from Ref. 61 with permission).

(a)

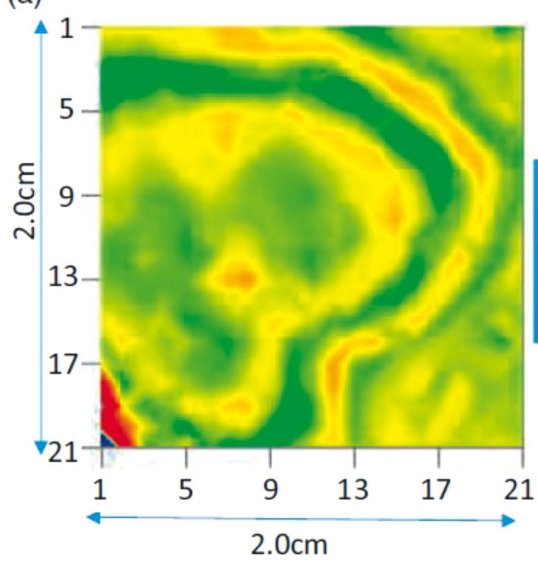

(b)

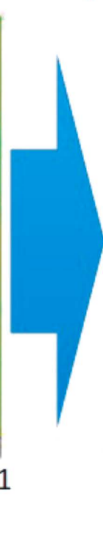

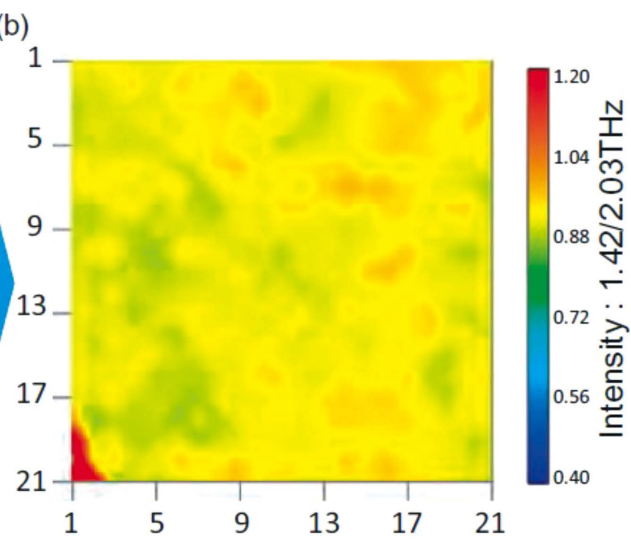

Fig. 23 The THz images of (a) the film just after hot pressing and (b) after melting the PCL film at room temperature (Reproduced from Ref. 61 with permission).

correlated to the degree of crystallinity of PCL. Intermolecular hydrogen bonding is considered between the two zigzag structures in the PCL crystalline lattice. The peak at $1.42 \mathrm{THz}$ originates from the perpendicular polarisation to the $c$-axis (fibre axis), while that at $2.03 \mathrm{THz}$ arises from the parallel mode. Hence, the different temperature-dependent behaviours of these two peaks are ascribed to the intermolecular interactions in the PCL crystalline lattice.

Figure 22(a) and (b), respectively, display the visual image of a PCL film and the corresponding $\mathrm{THz}$ transmission image developed using $I_{1.42} / I_{2.03} \cdot{ }^{61}$ Note that the visual image shows a homogeneous film surface; however, the $\mathrm{THz}$ image exhibits significant variations in the $I_{1.42} / I_{2.03}$ value. It seems that the $\mathrm{THz}$ image reflects the crystallinity distribution in the PCL film. Sato et al. ${ }^{61}$ confirmed the variations in the crystallinity between points A and B (Fig. 22) by WAXD and DSC measurements of the film.

Figure 23(a) and (b) shows the $\mathrm{THz}$ images of the film just after hot pressing and after melting the PCL film at room temperature, respectively. ${ }^{61}$ It is very likely that the crystalline orientation induced by the stress during the hot pressing still remains in the first sample; however, the crystalline orientation disappears completely during the melting process. The image in Fig. 23(a) shows concentric stripes, indicating that crystalline orientation takes place during the pressing of PCL. The value of the peak intensity ratio suggests that the molecular chains in the crystalline lamellae are oriented in the stretching direction. Hence, it was found from the THz image that the PCL crystalline lamellae orient in the form of concentric circles during hot pressing. The THz image in Fig. 23 contains information about both the crystalline orientation and crystallinity of the PCL film. Thus, to extract information only about the orientation, Sato et al. ${ }^{61}$ obtained polarised $\mathrm{THz}$ images using the peak intensity of PCL press film at $1.42 \mathrm{THz}$ for $90^{\circ}(\perp)$ and $0^{\circ}(/ /)$. Figure 24 displays the corresponding result. ${ }^{61}$ The angle between a vertical line on the image and the electric field of the $\mathrm{THz}$ wave was set to $0^{\circ}$ and $90^{\circ}$. If there are some polarisation properties in the PCL hot-pressed film, the image should show some sort of pattern. The stripe patterns in Fig. 24 suggest that the crystalline orientation occurs because the polymer chains are stretched and crystallised during the film preparation.

This study demonstrated that $\mathrm{THz}$ imaging is useful for visualising the distribution of crystallinity and crystalline orientations in the semi-crystalline polymer film. This technique can also be used in the polymer industry. 


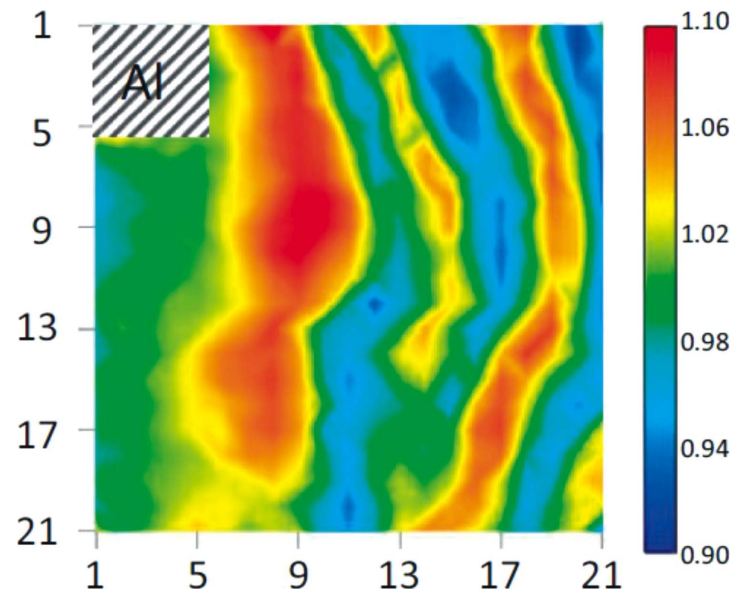

Fig. 24 The THz images of (a) the film just after hot pressing and (b) after melting the PCL film at room temperature (Reproduced from Ref. 61 with permission). advance. For analytical purposes, the applications of artificial intelligence, machine learning, and big data analysis are important.

In addition to the above three points, the development of instrumentation is particularly important; for example, further development of imaging techniques is required for MIR, NIR, and FIR or $\mathrm{THz}$ spectroscopy, in which 3D imaging is particularly noteworthy. Another important area is hand-held instruments; a combination of smartphone technology and portable imaging systems are also expected to advance. In the MIR region, nanoIR and cascade laser MIR spectroscopy are innovative spectroscopic techniques. For the NIR region, along with the miniaturisation of the instrument, laser-NIR spectroscopy is expected. In the FIR field, low-frequency VCD is promising.

In conclusion, I think the basic studies on MIR, NIR, and FIR or $\mathrm{THz}$ spectroscopy must be deepened more. Because of these studies, the applications of these three spectroscopic techniques will develop more widely.

\section{References}

1. (a) Y. Ozaki (ed.), "Advanced Vibrational Spectroscopyfrom Near-infrared to Terahertz", Anal. Sci., 2007, 23, 763 - 928. (b) T. Hasegawa and Y. Ozaki (ed.), "Frontiers of Vibrational Spectroscopy in Analytical Chemistry", Anal. Sci., 2017, 33, 1 - 123. (c) Y. Ozaki, Bull. Chem. Soc. Jpn., 2019, 92, 629.

2. P. R. Griffiths and J. A. de Haseth, "Fourier Transform Infrared Spectroscopy”, 2nd ed., 2007, Wiley Interscience, NJ.

3. (a) A. A. Christy, Y. Ozaki, and V. G. Gregoriou, "Modern Fourier Transform Infrared Spectroscopy", 2001, Elsevier, Amsterdam. (b) M. Tasumi (ed.), "Introduction to Experimental Infrared Spectroscopy: Fundamentals and Practical Methods", 2015, John Wiley \& Sons, Chichester, UK.

4. T. Hasegawa, "Quantitative Infrared Spectroscopy for Understanding of a Condensed Matter", 2017, Springer, Tokyo.

5. (a) H. W. Siesler, Y. Ozaki, S. Kawata, and H. M. Heise (ed.), "Near-Infrared Spectroscopy", 2002, Wiley-VCH, Weinheim. (b) Y. Ozaki, W. F. McClure, and A. A. Christy (ed.), "Near-Infrared Spectroscopy in Food Science and Technology", 2007, NJ.

6. Y. Ozaki, Anal. Sci., 2012, 28, 545.

7. Y. Ozaki, C. W. Huck, S. Tsuchikawa, and S. B. Engelsen (ed.), "Near-Infrared Spectroscopy: Theory, Spectral Analysis, Instrumentation, and Applications", 2020, Springer, Singapore.

8. K. D. Moller and W. G. Rothschield, "Far-Infrared Spectroscopy", 1971, Wiley Interscience, NJ.

9. A. Finch, "Chemical Applications of Far Infrared Spectroscopy", 1970, Academic Press, New York.

10. Y.-S. Lee, "Principle of Terahertz Science and Technology", 2009, Springer, New York.

11. S. L. Dexheimer, "Terahertz Spectroscopy: Principles and Applications", 2008, CRC Press, Boca Raton.

12. J. B. Baxter and G. W. Guglietta, Anal. Chem., 2011, 83 , 4342.

13. N. Sheppard, "The Historical Development of Experimental Techniques in Vibrational Spectroscopy", in "Handbook of Vibrational Spectroscopy", ed. J. M. Chalmers and P. R. Griffiths, 2002, Vol. 1, John Wiley \&x Sons, Chichester, $1-32$.

14. L. A. Nafie, "Vibrational Optical Activity, Principles and 
Applications", 2011, John Wiley \& Sons, Chichester.

15. (a) C. S. Peng, K. C. Jones, and A. Tokmakoff, J. Am. Chem. Soc., 2011, 133, 15650. (b) A. T. Krummel and M. T. Zanni, J. Phys. Chem. B, 2006, 110, 13991. (c) A. T. Krummel, P. Mukherjee, and M. T. Zanni, J. Phys. Chem. $B, \mathbf{2 0 0 3}, 107,9165$.

16. (a) C. Prater, K. Kjoller, D. Cook, R. Shetty, G. Meyers, C. Reinhardt, J. Felts, W. King, K. Vodopyanov, and A. Dazzi, Microsc. Anal., 2010, 24, 5. (b) K. Wieland, G. Ramer, V. U. Weiss, G. Allmaier, B. Lendl, and A. Centrone, Nano Res., 2019, 12, 197.

17. R. Salzer and H. W. Siesler, "Infrared and Raman Spectroscopic Imaging", 2nd ed., 2014, Wiley-VCH, Weinheim.

18. S. Šašić and Y. Ozaki, "Raman, Infrared, and Near-Infrared Chemical Imaging”, 2010, Wiley, NJ.

19. C. K. Akhgar, G. Ramer, M. Żbik, A. Trajnerowicz, J. Pawluczyk, A. Schwaighofer, and B. Lendl, Anal. Chem., 2020, 92, 9901.

20. T. Hasegawa and N. Shioya, Bull. Chem. Soc. Jpn., 2020, 93, 1127

21. (a) C. Sandrfy, "The Hydrogen Bond-Recent Development in Theory and Experiments", 1976, North-Holland. (b) C. Sandrfy, R. Buchet, and G. Lachenal, Ref. 5(b), 11.

22. (a) W. Kaye, Spectrochim. Acta, 1955, 7, 181. (b) O. H. Wheeler, Chem. Rev., 1959, 59, 629.

23. S. Cherdkeattikul, Y. Morisawa, and T. Ida, Anal. Sci., 2020, 36, 723.

24. (a) S.-W. Kuo, "Hydrogen Bonding in Polymeric Materials", 2018, Wiley-VCH, Weinheim. (b) P. E. Hansen, A. Jezierska, J. J. Panek, and J. Spanget-Larsen, "Theoretical Calculations are a Strong Tool in the Investigation of Strong Intramolecular Hydrogen Bonds" in "Molecular Spectroscopy; A Quantum Chemistry Approach", ed. Y. Ozaki, M. J. Wojcik, and J. Popp, 2019, Wiley-VCH, Weinheim, 215.

25. M. Saggu, N. M. Levinson, and S. G. Boxer, J. Am. Chem. Soc., 2011, 133, 17414.

26. M. Kazim, L. Guan, A. Chopra, R. Sun, M. A. Siegler, and T. Lectka, J. Org. Chem., 2020, 85, 981.

27. (a) H. Sato, R. Nakamura, A. Padermshoke, H. Yamaguchi, H. Terauchi, S. Ekgasit, I. Noda, and Y. Ozaki, Macromolecules, 2004, 37, 3764. (b) H. Sato, R. Murakami, A. Padermshoke, F. Hirose, K. Senda, I. Noda, and Y. Ozaki, Macromolecules, 2004, 37, 7203. (c) Y. Hu, J. Zhang, H. Sato, Y. Futami, I. Noda, and Y. Ozaki, Macromolecules, 2006, 39, 3841. (d) S. Yamamoto, Y. Morisawa, H. Sato, H. Hoshina, and Y. Ozaki, J. Phys. Chem. B, 2013, 117, 2180.

28. (a) H. Sato, Y. Ando, J. Dybal, T. Iwata, I. Noda, and Y. Ozaki, Macromolecules, 2008, 41, 4305. (b) H. Sato, Y. Ando, H. Mitomo, and Y. Ozaki, Macromolecules, 2011, $44,2829$.

29. (a) H. Matsuura, H. Yoshida, M. Hieda, S. Y. Yamanaka, T. Harada, K. Shin-ya, and K. Ohno, J. Am. Chem. Soc., 2003 125, 13910. (b) H. Yoshida, T. Harada, T. Murase, K. Ohno, and H. Matsuura, J. Phys. Chem. A, 1997, 101, 1731.

30. H. Wang and K. Tashiro, Macromolecules, 2016, 49, 581.

31. (a) V. Barone (ed.), "Computational Strategies for Spectroscopy: From Small Molecules to Nano Systems", 2011, Wiley. (b) J. Baker, "Molecular Structure and Vibrational Spectra", in "Handbook of Computational Chemistry", ed. J. Leszczynski, 2011, Springer Science and Business Media.

32. (a) M. J. Wojcik, H. Nakatsuji, B. Kirtman, and Y. Ozaki, (ed.), "Frontiers of Quantum Chemistry", 2018, Springer Nature, Singapore. (b) Y. Ozaki, M. J. Wojcik, and J. Popp, "Molecular Spectroscopy; A Quantum Chemical Approach",
2019, Wiley-VCH, Weinheim.

33. H. Yoshida, K. Takeda, J. Okamura, A. Ehara, and H. Matsuura, J. Phys. Chem. A, 2002, 106, 3580.

34. (a) K. B. Beć, J. Grabska, Y. Ozaki, J. P. Hawranek, and C. W. Huck, J. Phys. Chem. A, 2017, 121, 1412. (b) K. B. Beć, J. Grabska, and Y. Ozaki, Ref. 20, 483. (c) K. B. Beć, J. Grabska, Y. Ozaki, J. P. Hawranek, and C. W. Huck, J. Phys. Chem. A, 2017, 121, 1412.

35. Y. Ozaki, K. B. Bec, Y. Morisawa, S. Yamamoto, I. Tanabe, C. W. Huck, and T. S. Hofer, Chem. Soc. Rev., 2021, DOI: 101039/d0cs01602k.

36. S. G. Kazarian and K. L. Chan, Appl. Spectrosc., 2010, 64, $135 \mathrm{~A}$.

37. H. Lu, S. G. Kazarian, and H. Sato, Macromolecules, 2020, 53, 9074.

38. C. G. Kirchler, R. Henn, J. Modl, F. Münzker, T. H. Baumgartner, F. Meischl, A. Kehle, G. K. Bonn, and C. W. Huck, Molecules, 2018, 23, 3072.

39. M. A. Czarnecki, Y. Morisawa, Y. Futami, and Y. Ozaki, Chem. Rev., 2015, 115, 9707.

40. Y. Morisawa and A. Suga, Spectrochim. Acta, 2018, 197, 121.

41. (a) Y. Futami, Y. Ozaki, Y. Hamada, M. J. Wójcik, and Y. Ozaki, Chem. Phys. Lett., 2009, 482, 320. (b) Y. Futami, Y. Ozaki, Y. Hamada, M. J. Wójcik, and Y. Ozaki, J. Phys. Chem. A, 2011, 115, 1194.

42. (a) T. Gonjo, Y. Futami, Y. Morisawa, M. J. Wójcik, and Y. Ozaki, J. Phys. Chem. A, 2011, 115, 9845. (b) M. J. Schuler, T. S. Hofer, Y. Morisawa, Y. Futami, C. W. Huck, and Y. Ozaki, Phys. Chem. Chem. Phys., 2020, 22, 13017.

43. (a) K. Yagi, M. Keceli, and S. J. Hirata, Chem. Phys., 2012, 137, 204118. (b) K. Yagi, in "Molecular Spectroscopy; A Quantum Chemical Approach”, ed. Y. Ozaki, M. J. Wojcik, and J. Popp, 2019, Wiley-VCH, Weinheim, 147 - 170.

44. K. B. Beć, J. Grabska, C. W. Huck, and Y. Ozaki, in "Molecular Spectroscopy; A Quantum Chemical Approach", ed. Y. Ozaki, M. J. Wojcik, and J. Popp, 2019, Wiley-VCH, Weinheim, $353-388$.

45. J. O. Jung and R. B. Gerber, J. Chem. Phys., 1996, 105, 10332.

46. D. A. Clabo, W. D. Allen, R. B. Remington, Y. Yamaguchi, and H. F. Schaefer III, Chem. Phys., 1988, 123, 187.

47. K. B. Beć Y. Futami, M. J. Wójcik, and Y. Ozaki, Phys. Chem. Chem. Phys., 2016, 18, 13666.

48. J. Grabska, K. B. Beć, M. Ishigaki, C. W. Huck, and Y. Ozaki, J. Phys. Chem. B, 2018, 122, 6931.

49. (a) K. B. Beć, Y. Futami, M. J. Wójcik, T. Nakajima, and Y. Ozaki, J. Phys. Chem. A, 2016, 120, 6170. (b) J. Grabska, K. B. Beć, Y. Ozaki, and C. W. Huck, J. Phys. Chem. A, 2017, 121, 1950. (c) J. Grabska, M. A. Czarnecki, K. B. Beć, and Y. Ozaki, J. Phys. Chem. A, 2017, 121, 7925. (d) J. Grabska, M. Ishigaki, K. B. Beć, M. J. Wójcik, and Y. Ozaki, J. Phys. Chem. A, 2017, 121, 3437.

50. D. Ishikawa, H. Shinzawa, T. Genkawa, and Y. Ozaki, Anal. Sci., 2014, 30, 143.

51. (a) D. Ishikawa, K. Murayama, K. Awa, T. Genkawa, M. Komiyama, S. G. Kazarian, and Y. Ozaki, Anal. Biochem., 2013, 405, 9401. (b) D. Ishikawa, T. Nishii, F. Mizuno, H. Sato, S. G. Kazarian, and Y. Ozaki, Appl. Spectrosc., 2013 , 67, 1441.

52. M. Unger, Y. Ozaki, and H. W. Siesler, Appl. Spectrosc., 2011, 65, 1051.

53. M. Ishigaki, P. Puangchit, Y. Yasui, A. Ishida, H. Hayashi, Y. Nakayama, H. Taniguchi, I. Ishimaru, and Y. Ozaki, Anal. Chem., 2018, 90, 5217. 
54. M. Ishigaki, Y. Yasui, M. Kajita, and Y. Ozaki, Anal. Chem., 2020, $92,8133$.

55. (a) M. Ishigaki, S. Kawasaki, D. Ishikawa, and Y. Ozaki, Sci. Rep., 2016, 6, 20066. (b) M. Ishigaki, T. Nishii, P. Puangchit, Y. Yasui, C. W. Huck, and Y. Ozaki, J. Biophotonics, 2017, 21, 1003.

56. H. Hoshina, S. Ishii, S. Yamamoto, Y. Morisawa, H. Sato, T. Uchiyama, Y. Ozaki, and C. Otani, IEEE Trans. Terahertz Sci. Technol., 2013, 248.

57. S. Yamamoto, M. Miyada, H. Sato, H. Hoshina, and Y. Ozaki, J. Phys. Chem. B, 2017, 121, 1128.

58. C. Funaki, S. Yamamoto, H. Hoshina, Y. Ozaki, and H. Sato, Polymer, 2018, 137, 245.
59. D. Marlina, Y. Park, H. Hoshina, Y. Ozaki, Y. M. Jung, and H. Sato, Anal. Sci., 2020, 36, 731 .

60. S. Yamamoto, E. Ohnishi, H. Sato, H. Hoshina, D. Ishikawa, and Y. Ozaki, J. Phys. Chem. B, 2019, 123, 5368.

61. C. Funaki, T. Toyouchi, H. Hoshina, Y. Ozaki, and H. Sato, Appl. Spectrosc., 2017, 71, 1537.

62. H. W. Siesler and K. Holland-Moritz, "Infrared and Raman Spectroscopy of Polymers", 1980, Marcel Dekker, New York.

63. (a) K. M. Sørensen, F. van den Berg, and S. B. Engelsen, "Near-Infrared Spectroscopy", ed. Y. Ozaki, C. Huck, S. Tsuchikawa, and S. B. Engelsen, 2021, Chap. 7, Springer, 127. (b) S. Morita, Anal. Sci., 2020, 36, 107. 